\title{
Light pseudoscalar meson masses under strong magnetic fields within the SU(3) Nambu-Jona-Lasinio model
}

\author{
Sidney S. Avancini $\odot^{*}$ and Joana C. Sodré $\odot^{\dagger}$ \\ Departamento de Física, Universidade Federal de Santa Catarina, \\ 88040-900 Florianópolis, Santa Catarina, Brazil \\ Máximo Coppola $\odot^{\ddagger}$ and Norberto N. Scoccola ${ }^{\S}$ \\ CONICET, Rivadavia 1917, (1033) Buenos Aires, Argentina and Physics Department, \\ Comisión Nacional de Energía Atómica, Av. Libertador 8250, (1429) Buenos Aires, Argentina
}

(Received 10 September 2021; accepted 19 October 2021; published 29 November 2021)

\begin{abstract}
We calculate the pole masses of pseudoscalar mesons in a strongly magnetized medium within the framework of the SU(3) Nambu-Jona-Lasinio model, using a magnetic field-independent regularization scheme. We employ both a constant and a magnetic field-dependent coupling $G(B)$, the latter being fitted to reproduce lattice QCD results for the pseudocritical chiral transition temperature. Numerical results for the pole masses are obtained for definite parametrizations of the model. For neutral mesons, the use of $G(B)$ provides closer agreement with lattice QCD results, which reveal a decrease of the mass with the external field. On the contrary, charged mesons masses are enhanced by $B$, showing no sign of the nonmonotonous behavior found in recent lattice QCD simulations.
\end{abstract}

DOI: 10.1103/PhysRevD.104.094040

\section{INTRODUCTION}

The behavior of strongly interacting matter under the influence of intense magnetic fields has been attracting a lot of interest in recent years. This interest is partly motivated by the fact that strong magnetic fields have been achieved or hypothesized in several physical situations. For example, in noncentral relativistic heavy-ion collisions, magnetic fields of magnitude as large as $B \sim 10^{19}-10^{20} \mathrm{G}$ are generated due to the motion of charged spectator particles, essentially at the earliest times of the collision [1]. In addition, strong magnetic fields may also play an important role in astrophysics scenarios, such as matter formation in the early Universe [2] or in the dynamics of magnetars, where the inner core can possibly harbor magnetic fields strengths as large as $B \sim 10^{19} \mathrm{G}$ [3]. One expects that new and detectable effects in the phase diagram and properties of strongly interacting matter will emerge due to these extreme magnetic fields, causing numerous phenomenological consequences. For example,

\footnotetext{
*sidney.avancini@ufsc.br

joana.sodre@gmail.com

*coppola@tandar.cnea.gov.ar

scoccola@tandar.cnea.gov.ar
}

Published by the American Physical Society under the terms of the Creative Commons Attribution 4.0 International license. Further distribution of this work must maintain attribution to the author(s) and the published article's title, journal citation, and DOI. Funded by SCOAP ${ }^{3}$. the chiral magnetic effect [4-6], chiral separation effect [7], chiral magnetic wave [8-12], and related phenomena are supposed to be experimental signals of the influence of strong magnetic fields in the QCD matter [13,14].

From the theoretical point of view, first principle analytical QCD calculations are very difficult to perform in the nonperturbative regime given the complexity of the theory. Therefore, one has to make use of alternative procedures to tackle the problem. In this regard, great progress has been made in recent years on the investigation of the QCD phase diagram by using either lattice QCD (LQCD) simulations or effective models, which can work together in a complementary manner in the face of lack of experimental evidence in some observables. In fact, in many of these models available experimental or LQCD results are used to fix their phenomenological parameters, allowing for improved results. In particular, results from LQCD calculations at zero temperature and physical pion masses show that an external constant magnetic field enforces the quark condensate favoring the breakdown of chiral symmetry, an effect known as magnetic catalysis (MC) [15]. This result is in agreement with most effective model calculations [16,17]. At finite temperature, the majority of these models predict the increase of the pseudocritical transition temperature $T_{\mathrm{pc}}$ with the magnetic field $[16,17]$. Nevertheless, accurate LCQD results [18-21] have shown the opposite pattern; $T_{\mathrm{pc}}$ decreases with the magnetic field, a phenomenon dubbed as inverse magnetic catalysis (IMC). The explanation for IMC at finite 
temperature is still controversial and under study [see Ref. [22] for a minireview on the IMC effect]. It is not clear that the nonmonotic behavior of the quark condensate is in fact the mechanism behind the IMC effect [23-25]. In the context of effective models, several possibilities have been explored in the recent literature to incorporate the IMC effect phenomenologically. Within the Nambu-JonaLasinio (NJL) model for example, which we will use in this work, these improvements include going beyond meanfield calculations [26] or taking into consideration the anomalous magnetic moment of quarks [27-30]. Motivated by the running of the QCD coupling, one of the simplest modifications available consists of introducing a coupling constant that depends on the magnetic field (and in some cases also on the temperature) and can be fixed by fitting some LQCD results, such as the quark condensate or the chiral pseudocritical temperature. This strategy has shown that the NJL model can satisfactorily reproduce LQCD results in a broad range of temperature and magnetic fields [31-36]. In this regard, an interesting possibility was recently proposed in Ref. [37]. There, the magnetic field dependent four fermion coupling is fitted to reproduce constituent quark masses, which are obtained from the LQCD calculation of baryon masses by assuming in a simplified way that the baryon mass can be obtained by merely summing the masses of their constituents. Lastly, calculations using the nonlocal NJL model have shown that IMC is obtained naturally [38,39].

The presence of strong magnetic fields also has a meaningful impact on hadron properties. In this work we will focus on its consequences over the masses of the light pseudoscalar meson nonet, which has drawn a lot of attention in recent years. Most calculations in the literature have been performed for the lightest mesons. For pions at zero temperature, LQCD simulations show an overall decrease of the neutral pion mass with the magnetic field (both in quenched QCD and using staggered fermions), while charged pions exhibit the opposite behavior within the quenched approximation [40,41]. A recent simulation using highly improved staggered fermions with a slightly heavier-than-physical pion mass of $220 \mathrm{MeV}$ was performed in Ref. [42], where the masses of many pseudoscalar mesons are computed. There, the decreasing trend of the neutral pion (and kaon) mass is confirmed. Moreover, charged pions (and kaons) reveal an initial increase with the magnetic field up to values $e B \sim 0.6 \mathrm{GeV}^{2}$, in accordance with previous results from Ref. [18] where $e B<0.5 \mathrm{GeV}^{2}$ values are considered for the charged pion mass using stout smeared staggered fermions. However, in stark contrast with previous quenched results from Refs. [40,41], for stronger magnetic fields this increasing tendency is found to be reversed, resulting in a nonmonotonous behavior.

On the other hand, the influence of magnetic fields on the lightest scalar and pseudoscalar mesons $(\sigma$ and $\pi$ ) has also been calculated mostly using two-flavor schemes, such as chiral perturbation theory [43-46], the linear sigma model [47-49], two-flavor quark-meson model [50], relativistic Hamiltonian-based formalisms [45,51], effective chiral confinement Lagrangian approach [52,53], QCD sum rules [54], the two-flavor NJL model [34,55-64], or its nonlocal version $[65,66]$. In this context, there are very few calculations of meson properties incorporating the strange quark. In Refs. [67,68], using a nonrelativistic constituent SU(3) quark model, neutral and charged mesons masses are considered. By using a relativistic Hamiltonian-based formalism, in Refs. [45,51] pions and kaons are calculated and comparisons with chiral perturbation theory and LQCD results are considered. In Ref. [69], kaons and antikaons are investigated in a chiral SU(3) model.

In order to study the behavior of the masses of the light pseudoscalar meson nonet in the presence of an external constant magnetic field, we will use the SU(3) NJL model. We remind that the NJL model is a nonrenormalizable model and a regularization procedure has to be adopted, which may be considered as part of the definition of the effective model. In fact, the choice of an appropriate regularization scheme is a crucial issue for the description of physical systems. It has been shown that the use of an inappropriate regularization scheme causes strong oscillations in meson masses and tachyonic or discontinuous behavior of masses. When working with quark matter immersed in a magnetized medium, performing an exact separation of magnetic from nonmagnetic contributions for all physical observables is a key point for their correct description, a strategy known as the "magnetic field independent regularization" (MFIR) scheme. The importance of the regularization procedure has been reviewed in Ref. [70], where it is shown that the MFIR scheme is free of these unphysical behaviors, which are due to an improper regularization. An improvement within the MFIR scheme was recently suggested in Ref. [36] for the calculation of many mean-field observables. However, this modification is not relevant for the quantities we will study in this work, namely quark condensates and meson masses, so we can safely omit it.

Regarding the determination of meson masses within the NJL model, one important point is the proper calculation of charged mesons. In this case, polarization functions have to be carefully handled in order to be diagonalized, since Schwinger phases arising from quarks propagators do not cancel, leading to a breakdown of translational invariance. As shown in Ref. [58], an appropriate treatment involves the use of the Ritus basis [71]. Unfortunately, this issue has not been properly addressed in several calculations. Of course, for neutral mesons the usual momentum basis can be used since the Schwinger phase factor cancels out in that case. In this regard, the NJL model shows an enhancement of the neutral pion mass for sufficiently strong magnetic fields, in contradiction with lattice results. One possible approach to overcome this issue, which we will adopt in this work, is to introduce a magnetic field dependent 
coupling constant, determined by fitting LQCD results for the quark condensate, which, as mentioned earlier, also allows the model to incorporate the IMC effect at finite temperatures. Results obtained following this strategy agree very well with LQCD simulations [34,57]. An alternative approach comprises the use of the nonlocal version of the SU(2) NJL model, where the neutral pions mass has been shown to naturally decrease with the magnetic field in agreement with LQCD results $[65,66]$.

As mentioned earlier, there are very few calculations of meson properties incorporating the strange quark. The aim of the present paper is to study the behavior of the pseudoscalar meson nonet masses as functions of the magnetic field. To that end we use the SU(3) NJL model including the 't Hooft-Maekawa interaction which breaks the $\mathrm{U}_{\mathrm{A}}(1)$ symmetry. We work within the MFIR scheme and consider both the case of a fixed and of a $B$-dependent four-fermion coupling constant. At the mean-field level we calculate quark condensates, which are compared with LCQD results to find qualitative agreement. For the calculation of the light pseudoscalar meson nonet we adopt the RPA formulation, where special care has to be taken to the fact that constituent quark masses are different for each flavor. For charged mesons the Ritus basis is used to diagonalize the polarizers, resulting in monotonically increasing masses for both constant and magnetic couplings. For neutral mesons, the polarizers calculation is simplified since Schwinger phases cancels out. Note that the 't Hooft-Maekawa interaction together with the uniform magnetic field $B$ induce a mix of neutral mesons states with equal flavors, i.e., $\pi^{0}, \eta, \eta^{\prime}$ [72]. We see that, except for $\eta^{\prime}$, neutral pseudoscalar mesons display a nonmonotonous behavior when using a constant coupling, which shifts to a monotonous decrease in concordance with LCQD results when a magnetic coupling is introduced. As already known from the usual $\mathrm{SU}(3) \mathrm{NJL}$ model at $B=0$, the $\eta^{\prime}$ meson comes out in the model as a resonance or unstable particle. In this case, the propagator becomes a complex number and from the analysis of the complex pole, the mass of the resonance is obtained. This situation gets intricate when the magnetic field is present, thus, we have developed a new formalism to treat this case.

We organize this work as follows. In Sec. II we introduce the theoretical formalism used to obtain neutral and charged pseudoscalar meson masses. Then, in Sec. III we present and discuss our numerical results, while in Sec. IV we provide a summary of our work, together with our main conclusions. We also include Appendixes A and B to quote some technical details of our calculations.

\section{THEORETICAL FORMALISM}

\section{A. Effective Lagrangian and mean field properties}

We consider the Euclidean action of the SU(3) NJL model which includes a scalar-pseudoscalar interaction and the 't Hooft six-fermion interaction in the presence of an external magnetic field. It is written as

$$
\begin{aligned}
S_{E} & =\int d^{4} x\left[\bar{\psi}(-i \not D+\hat{m}) \psi-G \sum_{a=0}^{8}\left[\left(\bar{\psi} \lambda_{a} \psi\right)^{2}+\left(\bar{\psi} i \gamma_{5} \lambda_{a} \psi\right)^{2}\right]\right. \\
& \left.+K\left(d_{+}+d_{-}\right)\right]
\end{aligned}
$$

where $G$ and $K$ are coupling constants, $\psi=\left(\psi_{u}, \psi_{d}, \psi_{s}\right)^{T}$ represents a quark field with three flavors, $d_{ \pm}=$ $\operatorname{det}\left[\bar{\psi}\left(1 \pm \gamma_{5}\right) \psi\right]$ and $\hat{m}=\operatorname{diag}\left(m_{u}, m_{d}, m_{s}\right)$ is the corresponding current quark mass matrix. In addition, $\lambda_{0}=\sqrt{2 / 3} I$, where $I$ is the unit matrix in the three flavor space, and $\lambda_{a}$ with $a=1, \ldots, 8$ denote the Gell-Mann matrices. The coupling of quarks to the electromagnetic field $\mathcal{A}_{\mu}$ is implemented through the covariant derivative $D_{\mu}=\partial_{\mu}-i \hat{Q} \mathcal{A}_{\mu}$ where $\hat{Q}=\operatorname{diag}\left(Q_{u}, Q_{d}, Q_{s}\right)$ represents the quark electric charge matrix with $Q_{u} / 2=-Q_{d}=$ $-Q_{s}=e / 3$, e being the proton electric charge. In the present work we consider a static and constant magnetic field in the 3 direction. Using the Landau gauge we have $\mathcal{A}_{\mu}=\delta_{\mu 2} x_{1} B$.

In order to study meson properties, we proceed by bosonizing the action in terms of scalar $\sigma_{a}(x)$ and pseudoscalar $\pi_{a}(x)$ fields and the corresponding auxiliary $\mathrm{s}_{a}(x)$ and $\mathrm{p}_{a}(x)$ fields. Following the standard procedure, we start with the partition function

$$
Z=\int D \bar{\psi} D \psi e^{-S_{E}}
$$

By introducing functional delta functions, the scalar $\left(\bar{\psi} \lambda_{a} \psi\right)$ and pseudoscalar $\left(\bar{\psi} i \gamma_{5} \lambda_{a} \psi\right)$ terms present in $S_{E}$ are replaced by $\mathrm{s}_{a}(x)$ and $\mathrm{p}_{a}(x)$ and the functional integration on the fermionic fields $\psi$ and $\bar{\psi}$ can be performed by standard methods. To perform the integration over the auxiliary fields we use the stationary phase approximation (SPA), choosing $\tilde{\mathrm{s}}_{a}(x)$ and $\tilde{\mathrm{p}}_{a}(x)$ in order to minimize the integrand of the partition function. This yields a set of coupled equations among the bosonic fields; at the end, $\tilde{\mathrm{s}}_{a}(x)$ and $\tilde{\mathrm{p}}_{a}(x)$ are to be considered as implicit functions of $\sigma_{a}(x)$ and $\pi_{a}(x)$. Finally, we use the mean field approximation by expanding the bosonized action in powers of field fluctuations around the corresponding translationally invariant mean field values $\bar{\sigma}_{a}$ and $\bar{\pi}_{a}$, i.e., $\sigma_{a}(x)=$ $\bar{\sigma}_{a}+\delta \sigma_{a}(x)$ and $\pi_{a}(x)=\bar{\pi}_{a}+\delta \pi_{a}(x)$. Due to charge conservation, only $\bar{\sigma}_{0}, \bar{\sigma}_{3}$, and $\bar{\sigma}_{8}$ are different from zero, while the vacuum expectation values of pseudoscalar boson fields are zero, $\bar{\pi}_{a}=0$. For convenience, we introduce $\bar{\sigma}=\operatorname{diag}\left(\bar{\sigma}_{u}, \bar{\sigma}_{d}, \bar{\sigma}_{s}\right)=\lambda_{0} \bar{\sigma}_{0}+\lambda_{3} \bar{\sigma}_{3}+\lambda_{8} \bar{\sigma}_{8}$. At the mean field level, the Euclidean action per unit volume reads 


$$
\begin{aligned}
\frac{\bar{S}_{E}^{\text {bos }}}{V^{(4)}}= & -\frac{N_{c}}{V^{(4)}} \sum_{f=u, d, s} \int d^{4} x d^{4} x^{\prime} \operatorname{tr}_{D} \ln \left(\mathcal{S}_{x, x^{\prime}}^{f}\right)^{-1} \\
& -\frac{1}{2}\left[\bar{\sigma}_{f} \overline{\mathrm{s}}_{f}+G \overline{\mathrm{s}}_{f} \overline{\mathrm{s}}_{f}-\frac{K}{2} \overline{\mathrm{s}}_{u} \overline{\mathrm{s}}_{d} \overline{\mathrm{s}}_{s}\right]
\end{aligned}
$$

where $\operatorname{tr}_{D}$ stands for the trace in Dirac space while $\left(\mathcal{S}_{x, x^{\prime}}^{f}\right)^{-1}=\delta\left(x-x^{\prime}\right)\left[-i\left(\not \partial-i Q_{f} \not \mathcal{A}\right)+M_{f}\right]$ represents the inverse mean field quark propagator for each flavor with effective mass $M_{f}=m_{f}+\bar{\sigma}_{f}$. Moreover, $\overline{\mathrm{s}}_{f}=\tilde{\mathrm{s}}_{f}\left(\bar{\sigma}_{a}\right)$ represent the auxiliary fields at the mean field level within the SPA approximation (note that $\overline{\mathrm{p}}_{f}=0$ ). From the condition $\delta \bar{S}_{E}^{\text {bos }} / \delta \bar{\sigma}_{f}=0$ it follows that $\overline{\mathrm{s}}_{f}=2 \phi_{f}$, where $\phi_{f}$ is the chiral condensate for each flavor given by

$$
\phi_{f}=\left\langle\bar{\psi}_{f} \psi_{f}\right\rangle=-\frac{\delta \bar{S}_{E}^{\text {bos }}}{\delta m_{f}}=-\frac{N_{c}}{V^{(4)}} \int d^{4} x \operatorname{tr}_{D} \mathcal{S}_{x, x}^{f}
$$

As is well known, the quark propagator can be written in different ways [16,17]. For convenience we take the following one

$$
\mathcal{S}_{x, x^{\prime}}^{f}=e^{i \Phi_{f}\left(x, x^{\prime}\right)} \int_{p} e^{i p\left(x-x^{\prime}\right)} \tilde{\mathcal{S}}_{p}^{f}
$$

where $\Phi_{f}\left(x, x^{\prime}\right)=Q_{f} B\left(x_{1}+x_{1}^{\prime}\right)\left(x_{2}-x_{2}^{\prime}\right) / 2$ is the socalled Schwinger phase. We have introduced here the shorthand notation

$$
\int_{p} \equiv \int \frac{d^{4} p}{(2 \pi)^{4}}
$$

We express $\tilde{\mathcal{S}}_{p}^{f}$ in the Schwinger form $[16,17]$

$$
\begin{aligned}
\tilde{\mathcal{S}}_{p}^{f}= & \int_{0}^{\infty} d \tau \exp \left[-\tau\left(M_{f}^{2}+p_{\|}^{2}+\frac{\tanh \left(\tau B_{f}\right)}{\tau B_{f}} p_{\perp}^{2}-i \epsilon\right)\right] \\
& \times\left\{\left(M_{f}-p_{\|} \cdot \gamma_{\|}\right)\left[1+i s_{f} \gamma_{1} \gamma_{2} \tanh \left(\tau B_{f}\right)\right]-\frac{p_{\perp} \cdot \gamma_{\perp}}{\cosh ^{2}\left(\tau B_{f}\right)}\right\},
\end{aligned}
$$

where the following definitions have been used. The "perpendicular" and "parallel" gamma matrices are collected in vectors $\gamma_{\perp}=\left(\gamma_{1}, \gamma_{2}\right)$ and $\gamma_{\|}=\left(\gamma_{3}, \gamma_{4}\right)$. Similarly, $p_{\perp}=\left(p_{1}, p_{2}\right)$ and $p_{\|}=\left(p_{3}, p_{4}\right)$. Note that in our convention $\left\{\gamma_{\mu}, \gamma_{\nu}\right\}=-2 \delta_{\mu \nu}$ and we have introduced the notation $s_{f}=\operatorname{sign}\left(Q_{f} B\right)$ and $B_{f}=\left|Q_{f} B\right|$. The limit $\epsilon \rightarrow 0$ is implicitly understood.

The integral in Eq. (7) is divergent and has to be properly regularized. We will use the MFIR scheme, where one subtracts from the unregulated integral the $B=0$ limit and then adds it in a regulated form. We obtain

$$
\phi_{f}^{\mathrm{reg}}=\phi_{f}^{\mathrm{vac}}+\phi_{f}^{\mathrm{mag}}, \quad\left\{\begin{array}{l}
\phi_{f}^{\mathrm{vac}} \equiv-N_{c} M_{f} I_{1 f}^{\mathrm{vac}} \\
\phi_{f}^{\mathrm{mag}} \equiv-N_{c} M_{f} I_{1 f}^{\mathrm{mag}}
\end{array} .\right.
$$

The expression of $I_{1 f}^{\mathrm{vac}}$ for the $3 \mathrm{D}$ cutoff regularization scheme we use in this work can be found in Eq. (A5) of Appendix A. The expression of $I_{1 f}^{\mathrm{mag}}$, given in Eq. (B5) of Appendix B, reads

$I_{1 f}^{\mathrm{mag}}=\frac{B_{f}}{2 \pi^{2}}\left[\ln \Gamma\left(x_{f}\right)-\left(x_{f}-\frac{1}{2}\right) \ln x_{f}+x_{f}-\frac{\ln 2 \pi}{2}\right]$,

where $x_{f}=M_{f}^{2} /\left(2 B_{f}\right)$.

Finally, by combining the equations from the SPA together with the gap equations, we obtain that the regularized form of the set of coupled equations for the effective quarks masses read

$$
\begin{aligned}
& M_{u}=m_{u}-4 G \phi_{u}^{\mathrm{reg}}+2 K \phi_{d}^{\mathrm{reg}} \phi_{s}^{\mathrm{reg}}, \\
& M_{d}=m_{d}-4 G \phi_{d}^{\mathrm{reg}}+2 K \phi_{s}^{\mathrm{reg}} \phi_{u}^{\mathrm{reg}}, \\
& M_{s}=m_{s}-4 G \phi_{s}^{\mathrm{reg}}+2 K \phi_{u}^{\mathrm{reg}} \phi_{d}^{\mathrm{reg}}
\end{aligned}
$$

\section{B. Meson sector}

For the calculation of meson masses, we consider the second-order correction to the mean field bosonized Euclidean action $S_{E}$. At the quadratic level we get for the pseudoscalar sector

$S_{\text {mes }}^{\text {quad }}=\frac{1}{2} \int d^{4} x^{\prime} d^{4} x \sum_{P, P^{\prime}} \delta P^{*}(x) \mathcal{G}_{P, P^{\prime}}\left(x, x^{\prime}\right) \delta P^{\prime}\left(x^{\prime}\right)$,

where the sum indexes run over the nonet of pseudoscalar mesons. Namely, $P, P^{\prime}=\pi_{3}, \pi^{ \pm}, K^{0}, \bar{K}^{0}, K^{ \pm}, \eta_{0}, \eta_{8}$. The inverse meson propagator in coordinate space can be written as

$$
\mathcal{G}_{P, P^{\prime}}\left(x, x^{\prime}\right)=T_{P, P^{\prime}} \delta^{(4)}\left(x-x^{\prime}\right)-J_{P, P^{\prime}}\left(x, x^{\prime}\right) .
$$

For $P, P^{\prime}=\pi^{ \pm}, K^{ \pm}, K^{0}, \bar{K}^{0}$ this operator is diagonal

$T_{P, P^{\prime}}=T_{P} \delta_{P, P^{\prime}}, \quad J_{P, P^{\prime}}\left(x, x^{\prime}\right)=J_{P}\left(x, x^{\prime}\right) \delta_{P, P^{\prime}}$,

where

$$
\begin{aligned}
T_{\pi^{+}} & =T_{\pi^{-}}=\left[2 G-K \phi_{s}\right]^{-1}, \\
J_{\pi^{+}}\left(x, x^{\prime}\right) & =J_{\pi^{-}}\left(x^{\prime}, x\right)=c_{u d}\left(x, x^{\prime}\right), \\
T_{K^{+}} & =T_{K^{-}}=\left[2 G-K \phi_{d}\right]^{-1}, \\
J_{K^{+}}\left(x, x^{\prime}\right) & =J_{K^{-}}\left(x^{\prime}, x\right)=c_{u s}\left(x, x^{\prime}\right), \\
T_{K^{0}} & =T_{\bar{K}^{0}}=\left[2 G-K \phi_{u}\right]^{-1}, \\
J_{K^{0}}\left(x, x^{\prime}\right) & =J_{\bar{K}^{0}}\left(x^{\prime}, x\right)=c_{d s}\left(x, x^{\prime}\right) .
\end{aligned}
$$


In these expressions

$$
c_{f f^{\prime}}\left(x, x^{\prime}\right)=2 N_{c} \operatorname{tr}_{D}\left[\mathcal{S}_{x, x^{\prime}}^{f} \gamma_{5} \mathcal{S}_{x^{\prime}, x}^{f^{\prime}} \gamma_{5}\right] .
$$

On the other hand, the two-point function $\mathcal{G}_{P, P^{\prime}}\left(x, x^{\prime}\right)$ is nondiagonal but symmetric in the $P, P^{\prime}=\pi_{3}, \eta_{0}, \eta_{8}$ subspace. The corresponding matrix elements of $T_{P, P^{\prime}}$ are

$$
\begin{aligned}
T_{\pi_{3} \pi_{3}} & =\frac{K^{2}\left(\phi_{u}+\phi_{d}\right)^{2}-4 G K \phi_{s}-8 G^{2}}{f}, \\
T_{\eta_{0} \pi_{3}} & =\frac{2\left[K^{2}\left(\phi_{u}+\phi_{d}-\phi_{s}\right)-2 G K\right]\left(\phi_{u}-\phi_{d}\right)}{\sqrt{6} f}, \\
T_{\eta_{8} \pi_{3}} & =\frac{\left[K^{2}\left(\phi_{u}+\phi_{d}+2 \phi_{s}\right)+4 G K\right]\left(\phi_{u}-\phi_{d}\right)}{\sqrt{3} f}, \\
T_{\eta_{0} \eta_{0}} & =\frac{2 K^{2}\left[\left(\phi_{d}-\phi_{s}\right)^{2}+\phi_{u}\left(\phi_{u}-2 \phi_{d}-2 \phi_{s}\right)\right]+8 G K\left(\phi_{u}+\phi_{d}+\phi_{s}\right)-24 G^{2}}{3 f}, \\
T_{\eta_{8} \eta_{0}} & =\frac{2 K^{2}\left[\left(\phi_{u}-\phi_{d}\right)^{2}+\phi_{s}\left(\phi_{u}+\phi_{d}-2 \phi_{s}\right)\right]-4 G K\left(\phi_{u}+\phi_{d}-2 \phi_{s}\right)}{3 \sqrt{2} f}, \\
T_{\eta_{8} \eta_{8}} & =\frac{K^{2}\left[\left(\phi_{u}-\phi_{d}\right)^{2}+4 \phi_{s}\left(\phi_{u}+\phi_{d}+\phi_{s}\right)\right]-4 G K\left(2 \phi_{u}+2 \phi_{d}-\phi_{s}\right)-24 G^{2}}{3 f},
\end{aligned}
$$

where

$$
f=-4 K^{3} \phi_{u} \phi_{d} \phi_{s}+4 G K^{2}\left(\phi_{u}^{2}+\phi_{d}^{2}+\phi_{s}^{2}\right)-16 G^{3} .
$$

In turn, the polarization function elements can be expressed as

$$
J_{P, P^{\prime}}\left(x, x^{\prime}\right)=\sum_{f} \gamma_{P, P^{\prime}}^{f} c_{f f}\left(x, x^{\prime}\right),
$$

where the coefficients $\gamma_{P, P^{\prime}}^{f}$ are given by

$$
\begin{array}{lll}
\gamma_{\pi_{3} \pi_{3}}^{u}=+\gamma_{\pi_{3} \pi_{3}}^{d}=\frac{1}{2}, & \gamma_{\pi_{3} \pi_{3}}^{s}=0, & \gamma_{\eta_{0} \eta_{0}}^{u}=\gamma_{\eta_{0} \eta_{0}}^{d}=\gamma_{\eta_{0} \eta_{0}}^{s}=\frac{1}{3}, \\
\gamma_{\eta_{0} \pi_{3}}^{u}=-\gamma_{\eta_{0} \pi_{3}}^{d}=\frac{1}{\sqrt{6}}, & \gamma_{\eta_{0} \pi_{3}}^{s}=0, & \gamma_{\eta_{8} \eta_{0}}^{u}=\gamma_{\eta_{8} \eta_{0}}^{d}=-\frac{1}{2} \gamma_{\eta_{8} \eta_{0}}^{s}=\frac{1}{3 \sqrt{2}}, \\
\gamma_{\eta_{8} \pi_{3}}^{u}=-\gamma_{\eta_{8} \pi_{3}}^{d}=\frac{1}{2 \sqrt{3}}, & \gamma_{\eta_{8} \pi_{3}}^{s}=0, & \gamma_{\eta_{8} \eta_{8}}^{u}=\gamma_{\eta_{8} \eta_{8}}^{d}=\frac{1}{4} \gamma_{\eta_{8} \eta_{8}}^{s}=\frac{1}{6} .
\end{array}
$$

\section{Neutral mesons}

For neutral mesons the contributions of Schwinger phases associated with the quark propagators in Eq. (17) cancel out. Therefore, the polarization functions depend only on the difference $\left(x-x^{\prime}\right)$, which leads to the conservation of momentum, since they are translationally invariant. If we take the Fourier transform of neutral meson fields to the momentum basis, the corresponding transform of the polarization functions will be diagonal in momentum space. Thus, the neutral meson contribution to the quadratic action in the momentum basis can be written as

$$
\begin{aligned}
S_{\text {neut.mes }}^{\text {quad }}= & \frac{1}{2} \int_{q} \sum_{P=K^{0}, \bar{K}^{0}} \delta P^{*}(-q) \mathcal{G}_{P}\left(q_{\perp}^{2}, q_{\|}^{2}\right) \delta P(q) \\
& +\frac{1}{2} \int_{q} \sum_{P, P^{\prime}=\pi_{3}, \eta_{0}, \eta_{8}} \delta P^{*}(-q) \mathcal{G}_{P, P^{\prime}}\left(q_{\perp}^{2}, q_{\|}^{2}\right) \delta P^{\prime}(q) .
\end{aligned}
$$

Here, the inverse neutral kaon propagator is given by

$$
\begin{aligned}
\mathcal{G}_{K^{0}}\left(q_{\perp}^{2}, q_{\|}^{2}\right) & =\mathcal{G}_{\bar{K}^{0}}\left(q_{\perp}^{2}, q_{\|}^{2}\right) \\
& =\left[2 G-K \phi_{u}\right]^{-1}-c_{d s}\left(q_{\perp}^{2}, q_{\|}^{2}\right),
\end{aligned}
$$


while for $P, P^{\prime}=\pi_{3}, \eta_{0}, \eta_{8}$ we have

$$
\mathcal{G}_{P, P^{\prime}}\left(q_{\perp}^{2}, q_{\|}^{2}\right)=T_{P, P^{\prime}}+\sum_{f} \gamma_{P, P^{\prime}}^{f} c_{f f}\left(q_{\perp}^{2}, q_{\|}^{2}\right) .
$$

The values of $T_{P, P^{\prime}}$ and $\gamma_{P, P^{\prime}}^{f}$ can be found in Eqs. (18) and (21), respectively.

In the neutral case, the functions $c_{f f^{\prime}}$ in momentum space are given by

$$
c_{f f^{\prime}}\left(q_{\perp}^{2}, q_{\|}^{2}\right)=2 N_{c} \int_{p} \operatorname{tr}_{D}\left[\tilde{\mathcal{S}}_{p_{-}}^{f} \gamma_{5} \tilde{\mathcal{S}}_{p_{+}}^{f^{\prime}} \gamma_{5}\right]
$$

where $p^{ \pm}=p \pm q / 2$. We remark here that these functions are divergent. Within the MFIR scheme they can be regularized as

$$
c_{f f^{\prime}}^{\mathrm{reg}}\left(q_{\perp}^{2}, q_{\|}^{2}\right)=c_{f f^{\prime}}^{\mathrm{vac}}\left(q^{2}\right)+c_{f f^{\prime}}^{\mathrm{mag}}\left(q_{\perp}^{2}, q_{\|}^{2}\right)
$$

where the first term in the right-hand side correspond to the vacuum contribution while the second term to the magnetic one. In this work we regularize the otherwise divergent vacuum term through a 3D cutoff; the corresponding expression is given in Eq. (A13) of Appendix A. For the calculation of the masses we can set $q_{\perp}^{2}=0$, while the Euclidean parallel components are to be evaluated at the negative real space $q_{\|}^{2}=-m_{P}^{2}$, with $m_{P}>0$. Then, assuming that $m_{P}<M_{f}+M_{f^{\prime}}$, the magnetic contribution can be written as (see Appendix B)

$$
\begin{aligned}
& c_{f f^{\prime}}^{\mathrm{mag}}\left(0, q_{\|}^{2}=-m_{P}^{2}\right) \\
& \quad=2 N_{c}\left\{\frac{I_{1 f}^{\mathrm{mag}}+I_{1 f^{\prime}}^{\mathrm{mag}}}{2}-\left[m_{P}^{2}-\left(M_{f}-M_{f^{\prime}}\right)^{2}\right] I_{2 f f^{\prime}}^{\mathrm{mag}}\left(-m_{P}^{2}\right)\right\} .
\end{aligned}
$$

The function $I_{1 f}^{\mathrm{mag}}$ has already been expressed in Eq. (9) while

$$
I_{2 f f^{\prime}}^{\mathrm{mag}}\left(-m_{P}^{2}\right)=\frac{1}{8 \pi^{2}} \lim _{\epsilon \rightarrow 0} \int_{0}^{1} d y\left[\psi\left(\bar{x}_{f f^{\prime}}-i \epsilon\right)-\ln \left(\bar{x}_{f f^{\prime}}-i \epsilon\right)+\frac{1}{2\left(\bar{x}_{f f^{\prime}}-i \epsilon\right)}\right],
$$

where $\psi(x)$ is the digamma function and we have defined

$$
\bar{x}_{f f^{\prime}}=\frac{y M_{f}^{2}+(1-y) M_{f^{\prime}}^{2}-y(1-y) m_{P}^{2}}{2 B_{f}} .
$$

For $m_{P}<M_{f}+M_{f^{\prime}}$ we have that $\bar{x}_{f f^{\prime}}>0$ for all values of $y$ within the integration range of the integral of Eq. (28). Thus, the limit $\epsilon \rightarrow 0$ can be directly taken.

On the other hand, for $\eta^{\prime}$ we expect that $m_{P}>M_{f}+M_{f^{\prime}}$. In this case one has to have special care since $\bar{x}_{f f^{\prime}}$ can be negative within the interval $0<y<1$. We proceed by taking the analytic continuation of both the digamma and logarithm functions. This implies that the inverse propagators become complex functions. Thus, we assume that $q_{\|}$develops an imaginary part

$$
q_{\|}^{2}=-\left(m_{P}-\frac{i}{2} \Gamma_{P}\right)^{2}
$$

where $\Gamma_{P}$ is associated with the decay width of the meson. Following the customary method introduced in Ref. [73], we assume that the width is not too large and neglect its contribution inside $I_{2 f f^{\prime}}^{\mathrm{mag}}$ function (this also applies to the equivalent vacuum contribution)

$$
c_{f f^{\prime}}^{\mathrm{mag}}\left(m_{P}, \Gamma_{P}\right) \simeq 2 N_{c}\left\{\frac{I_{1 f}^{\mathrm{mag}}+I_{1 f^{\prime}}^{\mathrm{mag}}}{2}-\left[\left(m_{P}-\frac{i}{2} \Gamma_{P}\right)^{2}-\left(M_{f}-M_{f^{\prime}}\right)^{2}\right] I_{2 f f^{\prime}}^{\mathrm{mag}}\left(-m_{P}^{2}\right)\right\} .
$$

Note that in Eq. (28) one might hit some poles of the digamma function if the limit $\epsilon \rightarrow 0$ is naively taken. As detailed in Appendix B, through a careful treatment of these poles one can explicitly calculate the $I_{2 f f^{\prime}}^{\mathrm{mag}}$ function. The general result for $f \neq f^{\prime}$ is given in Eq. (B13) of Appendix B. We remark here that, as a consistency check, we have repeated the calculation using the Landau level representation of the quark propagator, well defined for all $m_{P}$, obtaining the same result. For the determination of the $\eta^{\prime}$ mass we only need the $f=f^{\prime}$ version of the general expression, given by

$$
\begin{aligned}
I_{2 f f}^{\mathrm{mag}}\left(-m_{P}^{2}\right)= & -\frac{1}{8 \pi^{2}}\left\{\ln \left(\frac{M_{f}^{2}}{2 B_{f}}\right)+2 \beta_{0} \ln \left[\frac{m_{P}\left(1+\beta_{0}\right)}{2 M_{f}}\right]-2+\frac{2 B_{f}}{m_{P}^{2}} \sum_{n=0}^{N} \frac{g_{n}}{\beta_{n}} \ln \left(\frac{1-\beta_{n}}{1+\beta_{n}}\right)\right\} \\
& +\frac{1}{8 \pi^{2}} \int_{0}^{1} d y \psi\left(\bar{x}_{f f}+N+1\right)+\frac{i}{8 \pi}\left[\beta_{0}-\frac{2 B_{f}}{m_{P}^{2}} \sum_{n=0}^{N} \frac{g_{n}}{\beta_{n}}\right]
\end{aligned}
$$


where $g_{n}=2-\delta_{n 0}$ and $N=$ Floor $\left[m_{P}^{2} \beta_{0}^{2} / 8 B_{f}\right]$. Moreover,

$$
\beta_{n}=\sqrt{1-\frac{4 M_{f}^{2}}{m_{P}^{2}}-\frac{8 n B_{f}}{m_{P}^{2}}} .
$$

For the neutral kaons, we expect $m_{K^{0}}=m_{\bar{K}^{0}}<$ $M_{d}+M_{s}$. In this case the polarization function is real and $I_{2 f f^{\prime}}^{\mathrm{mag}}$ is well defined in the $\epsilon \rightarrow 0$ limit of Eq. (28). Therefore, the pole mass will be given by the solution of

$$
\mathcal{G}_{K^{0}}\left(q_{\perp}^{2}=0, q_{\|}^{2}=-m_{K^{0}}^{2}\right)=0 .
$$

In the $P, P^{\prime}=\pi_{3}, \eta_{0}, \eta_{8}$ subspace, the corresponding quadratic action can be expressed in matrix notation through the following inverse matrix propagator

$$
\mathcal{M}=\left(\begin{array}{lll}
\mathcal{G}_{\pi_{3} \pi_{3}} & \mathcal{G}_{\pi_{3} \eta_{0}} & \mathcal{G}_{\pi_{3} \eta_{8}} \\
\mathcal{G}_{\eta_{0} \pi_{3}} & \mathcal{G}_{\eta_{0} \eta_{0}} & \mathcal{G}_{\eta_{0} \eta_{8}} \\
\mathcal{G}_{\eta_{8} \pi_{3}} & \mathcal{G}_{\eta_{0} \eta_{8}} & \mathcal{G}_{\eta_{8} \eta_{8}}
\end{array}\right)
$$

which is actually symmetric. The physical meson pole masses and widths will be given by the roots of

$$
\operatorname{det}\left[\mathcal{M}\left(m_{P}, \Gamma_{P}\right)\right]=0,
$$

where the three pair of roots are to be associated with the $\pi^{0}, \eta, \eta^{\prime}$. Of course, one expects to get $\Gamma_{\pi^{0}}=\Gamma_{\eta}=0$ while $\Gamma_{\eta^{\prime}}$ is expected to be nonvanishing. Note that when $B=0$, $\pi_{3}$ (and therefore $\pi^{0}$ ) decouples from the $\eta_{0}, \eta_{8}$ states due to isospin symmetry. However, in the presence of an external magnetic field this symmetry breaks down due to different quark electric charges. In this case, the $\pi^{0}, \eta, \eta^{\prime}$ neutral mesons consist of a mix of $\pi_{3}, \eta_{0}, \eta_{8}$ states, reflected by the fact that nondiagonal terms are present in the inverse propagator of Eq. (35).

\section{Charged mesons}

In this case the contributions of Schwinger phases associated with the quark propagators do not cancel out, leading to a breakdown of translational invariance. In order to diagonalize the charged meson fields, we employ the Ritus-like formalism. We find it convenient to introduce the following notation convention

$$
\begin{aligned}
\delta P(x) & =\bigvee_{\bar{q}} \mathbb{F}_{\bar{q}}(x) \delta P(\bar{q}), \\
\bigvee_{\bar{q}} & \equiv \frac{1}{(2 \pi)^{4}} \sum_{k=0}^{\infty} \int d q_{2} d q_{3} d q_{4},
\end{aligned}
$$

with $\bar{q}=\left(k, q_{2}, q_{3}, q_{4}\right)$ where $k$ labels the charged meson Landau level. The Ritus-like eigenfunctions are

$$
\mathbb{F}_{\bar{q}}^{P}(x)=N_{k} e^{i\left(q_{2} x_{2}+q_{3} x_{3}+q_{4} x_{4}\right)} D_{k}\left(\rho_{P}\right) .
$$

Here $D_{k}(x)$ are the cylindrical parabolic functions. We have also defined $N_{k}=\left(4 \pi B_{P}\right)^{1 / 4} / \sqrt{k !}$ and $\rho_{P}=$ $\sqrt{2 B_{P}} x_{1}-s_{P} \sqrt{2 / B_{P}} q_{2}$, where $B_{P}=\left|Q_{P} B\right|$ and $s_{P}=$ $\operatorname{sign}\left(Q_{P} B\right)$. Note that in our case, for the $\pi^{ \pm}$and $K^{ \pm}$ mesons these definitions reduce to $B_{P}=|e B|$ and $s_{\pi^{ \pm}}=s_{K^{ \pm}}= \pm 1$.

The corresponding transformed polarization functions will be diagonal in $\bar{q}, \bar{q}^{\prime}$ space. Thus, the charged meson contribution to the quadratic action in the Ritus basis can be written as

$$
S_{\text {char.mes }}^{\text {quad }}=\frac{1}{2} \bigvee_{\bar{q}} \sum_{P=\pi^{ \pm}, K^{ \pm}} \delta P^{*}(\bar{q}) \mathcal{G}_{P}\left(k, \Pi^{2}\right) \delta P(\bar{q}),
$$

where $\Pi^{2}=q_{\|}^{2}+(2 k+1) B_{P}$. The inverse propagators read

$$
\begin{aligned}
& \mathcal{G}_{\pi^{ \pm}}\left(k, \Pi^{2}\right)=\left[2 G-K \phi_{s}\right]^{-1}-c_{u d}\left(k, \Pi^{2}\right), \\
& \mathcal{G}_{K^{ \pm}}\left(k, \Pi^{2}\right)=\left[2 G-K \phi_{d}\right]^{-1}-c_{u s}\left(k, \Pi^{2}\right),
\end{aligned}
$$

where we have used

$$
\begin{aligned}
& \int d^{4} x^{\prime} d^{4} x\left[\mathbb{F}_{\bar{q}}^{\frac{\pi}{}}(x)\right]^{*} c_{u d}\left(x, x^{\prime}\right) \mathbb{F}_{\bar{q}^{\prime}}^{\pi^{+}}\left(x^{\prime}\right)=c_{u d}\left(k, \Pi^{2}\right)(2 \pi)^{4} \delta_{\bar{q}, \bar{q}^{\prime}}, \\
& \int d^{4} x^{\prime} d^{4} x\left[\mathbb{F}_{\bar{q}}^{K^{+}}(x)\right]^{*} c_{u s}\left(x, x^{\prime}\right) \mathbb{F}_{\bar{q}^{\prime}}^{K^{+}}\left(x^{\prime}\right)=c_{u s}\left(k, \Pi^{2}\right)(2 \pi)^{4} \delta_{\bar{q}, \bar{q}^{\prime}}
\end{aligned}
$$

These functions are divergent and need to be regularized. Within the MFIR scheme using a 3D cutoff they can be expressed as

$$
c_{f f^{\prime}}^{\mathrm{reg}}\left(k, \Pi^{2}\right)=c_{f f^{\prime}}^{\mathrm{vac}}\left(\Pi^{2}\right)+c_{f f^{\prime}}^{\mathrm{mag}}\left(k, \Pi^{2}\right) .
$$

The regularized vacuum contribution is given in Appendix A. After a long but straightforward calculation (see [59] for details), we obtain the following expression for the magnetic contribution 


$$
\begin{aligned}
c_{f f^{\prime}}^{\mathrm{mag}}\left(k, \Pi^{2}\right)= & \frac{N_{c}}{2 \pi^{2}} \int_{0}^{\infty} d z \int_{0}^{1} d y e^{-z\left[y M_{f}^{2}+(1-y) M_{f^{\prime}}^{2}+y(1-y) \Pi^{2}\right]} \\
& \times\left\{\left[M_{f} M_{f^{\prime}}+\frac{1}{z}-y(1-y)\left(\Pi^{2}-(2 k+1) B_{P}\right)\right]\left[\frac{\left(1+s_{f} S_{f^{\prime}} t_{f} t_{f^{\prime}}\right)}{\alpha_{+}}\left(\frac{\alpha_{-}}{\alpha_{+}}\right)^{k} e^{z y(1-y)(2 k+1) B_{P}}-\frac{1}{z}\right]\right. \\
& \left.+\frac{\left(1-t_{f}^{2}\right)\left(1-t_{f^{\prime}}^{2}\right)}{\alpha_{+}^{2} \alpha_{-}}\left(\frac{\alpha_{-}}{\alpha_{+}}\right)^{k}\left[\alpha_{-}+k\left(\alpha_{-}-\alpha_{+}\right)\right] e^{z y(1-y)(2 k+1) B_{P}}-\frac{1}{z}\left[\frac{1}{z}-y(1-y)(2 k+1) B_{P}\right]\right\},
\end{aligned}
$$

where we have introduced the definitions $t_{f}=\tanh \left(B_{f} z y\right)$, $t_{f^{\prime}}=\tanh \left[B_{f^{\prime}} z(1-y)\right]$ and $\alpha_{ \pm}=\left(B_{f^{\prime}} t_{f}+B_{f} t_{f^{\prime}} \pm B_{P} t_{f} t_{f^{\prime}}\right) /$ $\left(B_{f} B_{f^{\prime}}\right)$. Note also that $Q_{P}=Q_{f}-Q_{f^{\prime}}$.

For pions and kaons we expect them to develop only a real pole mass, i.e., $\Pi^{2}=-m_{P}^{2}$ with $m_{P}<M_{f}+M_{f^{\prime}}$. In that case the integrals in Eq. (43) are convergent and well defined. Therefore, for each Landau level the charged mesons pole masses will be given by the solutions of

$$
\begin{gathered}
\mathcal{G}_{\pi^{ \pm}}\left(k, \Pi^{2}=-m_{\pi^{ \pm}}^{2}\right)=0, \\
\mathcal{G}_{K^{ \pm}}\left(k, \Pi^{2}=-m_{K^{ \pm}}^{2}\right)=0 .
\end{gathered}
$$

\section{NUMERICAL RESULTS}

To obtain numerical results for the magnetic field dependence of the meson masses one has to fix the model parametrization. Here, following Ref. [73], we take the parameter set $m_{u}=m_{d}=5.5 \mathrm{MeV}, m_{s}=140.7 \mathrm{MeV}$, $\Lambda=602.3 \mathrm{MeV}, G \Lambda^{2}=1.835$, and $K \Lambda^{5}=12.36$, which has been determined on fixing that for vanishing external field one gets $m_{\pi}=135 \mathrm{MeV}, \quad m_{K}=497.7 \mathrm{MeV}$, $m_{\eta^{\prime}}=957.8 \mathrm{MeV}$, and $f_{\pi}=92.4 \mathrm{MeV}$. This parameter set gives an $\eta$ mass of $m_{\eta}=514.8 \mathrm{MeV}$, which compares reasonably well with the physical value $m_{\eta}^{p h s}=548.8 \mathrm{MeV}$, together with an appropriate value for the chiral condensate of $\left\langle\bar{\psi}_{f} \psi_{f}\right\rangle^{1 / 3}=242 \mathrm{MeV}$ for $f=u, d$. As mentioned in the Introduction, while local NJL-like models are able to reproduce the $\mathrm{MC}$ effect at vanishing temperature, they fail to lead to the IMC effect. Among the possible ways to deal with this problem, one of the simplest consists of allowing the model parameters to depend on the magnetic field. Motivated by this we also explore the possibility of considering a magnetic field dependent coupling $G(B)$. We adopt the one proposed in Ref. [31] in the context of an SU(3) NJL model with the same parameters that we use. In that work the current quark masses, $\Lambda$ and $K$ were kept constant while for $G(B)$ the form

$G(B)=G\left[\frac{1+a\left(e B / \Lambda_{\mathrm{QCD}}^{2}\right)^{2}+b\left(e B / \Lambda_{\mathrm{QCD}}^{2}\right)^{3}}{1+c\left(e B / \Lambda_{\mathrm{QCD}}^{2}\right)^{2}+d\left(e B / \Lambda_{\mathrm{QCD}}^{2}\right)^{4}}\right]$,

was introduced. Here, $a=0.0108805, b=-1.0133 \times 10^{-4}$, $c=0.02228, d=1.84558 \times 10^{-4}$, and $\Lambda_{\mathrm{QCD}}=300 \mathrm{MeV}$. As stated in Ref. [31], this form of the scalar coupling has been fitted so that the lattice QCD pseudocritical chiral transition temperatures are reproduced.

Results for the magnetic field dependence of the dynamical quark masses are shown in Fig. 1, for both constant and $B$-dependent coupling $G$. As we see, for constant $G$ all quark masses increase with $B$. In contrast, for $G(B)$ they display a nonmonotonous behavior, less affected by the magnetic field. In this case, $M_{d}$ and $M_{s}$ initially decrease with $B$, while about $e B \sim 0.6-0.7 \mathrm{GeV}^{2}$ this tendency reverses. On the other hand, $M_{u}$ has just the opposite behavior. In fact, these dependencies of the dynamical quark masses on the magnetic field are roughly consistent with the results obtained in Ref. [37]. In that work these quantities have been extracted from a LQCD calculation of the baryon masses using a simple minded approximation based on the constituent quark model.

It should be stressed that in spite of the rather different behavior between the dynamical quark masses, a magnetic catalysis effect at zero temperature is obtained independently on whether $G$ depends on $B$ or not. This is shown in Fig. 2, where we displayed the conveniently normalized light quark condensates. These quantities are defined as it follows. As in Ref. [19], for the case of vanishing temperature we are interested in, we first introduce

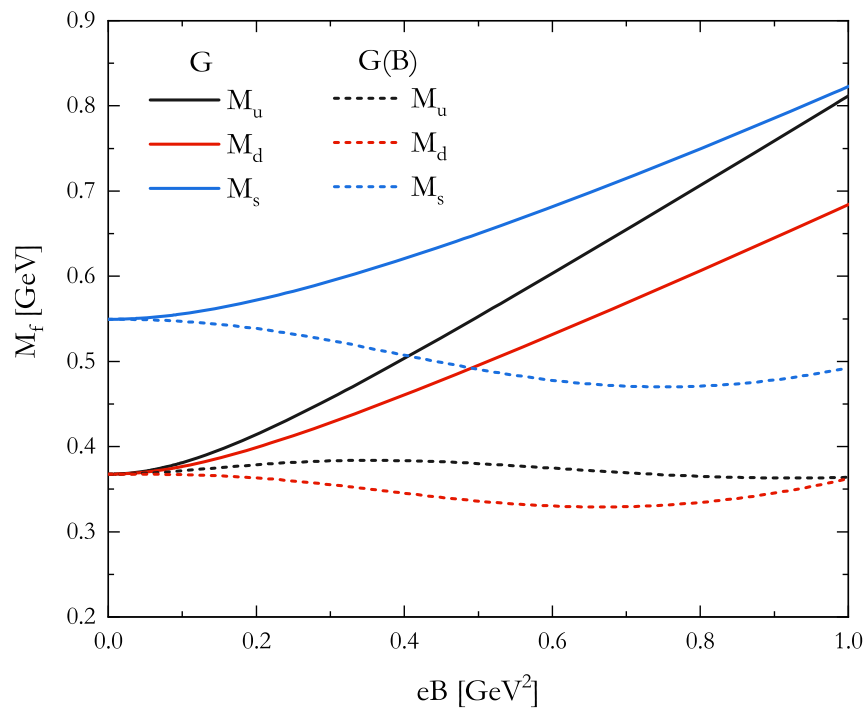

FIG. 1. Effective quark masses $M_{u}$ (black), $M_{d}$ (red), and $M_{s}$ (blue) as functions of $e B$ for fixed (solid lines) and $B$-dependent (dashed lines) coupling $G$. 

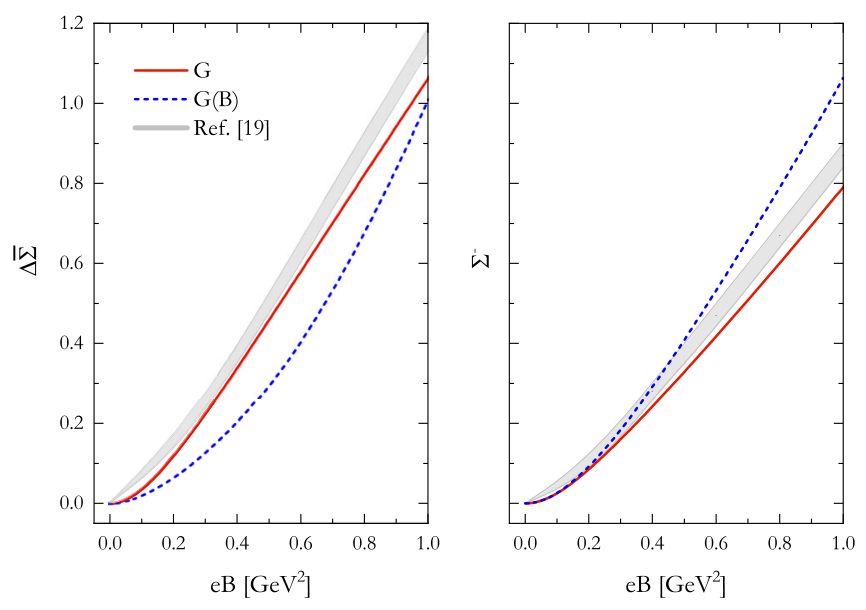

FIG. 2. Left: average condensate as a function of $e B$. Right: condensate difference as a function of $e B$. Results for constant (red solid lines) and $B$-dependent (blue dashed lines) coupling $G$ are shown. LQCD results from Ref. [19] (gray bands) are added for comparison.

$$
\Sigma_{f}=\frac{2 m_{f}}{D^{4}}\left[\phi_{f}^{\mathrm{reg}}(B)-\phi_{f}^{\mathrm{reg}}(0)\right]+1,
$$

where we have explicitly stated the magnetic field dependence of the quark condensate, defined in Eq. (8). Moreover, $D=(86 \mathrm{MeV} \times 135 \mathrm{MeV})^{1 / 2}$ was introduced in Ref. [19] as a kind of normalization constant and $m_{f}$ is the current quark mass of each light flavor. Then, in the left panel of Fig. 2 we plot $\Delta \bar{\Sigma}=\left(\Sigma_{u}+\Sigma_{d}\right) / 2-1$ while in the right panel the difference $\Sigma^{-}=\Sigma_{u}-\Sigma_{d}$ is shown. The gray bands in Fig. 2 correspond to LQCD results taken from Ref. [19], whereas full red (dashed blue) lines represent our results for constant $G$ ( $B$-dependent $G$ ). We observe that although the predictions for constant $G$ are somewhat closer to the LQCD results, those corresponding to $G(B)$ can certainly be considered as acceptable. It is interesting to remark here that other form functions of $G(B)$, such as the ones proposed in Refs. [34,37], reproduce similar trends for these quantities.

We turn now to our results for the magnetic field dependence of the masses of the nonet of pseudoscalar mesons. They are shown in Fig. 3, where for charged mesons we instead display their lowest energy states, given by

$$
\begin{aligned}
E_{P^{ \pm}} & =\left.\sqrt{m_{P^{ \pm}}^{2}+(2 k+1) e B+q_{3}^{2}}\right|_{\substack{q_{3}=0 \\
k=0}} \\
& =\sqrt{m_{P^{ \pm}}^{2}+e B},
\end{aligned}
$$

(note that both $E_{P}$ and $m_{P}$ depend on $B$ although not explicitly stated). The left (right) panel corresponds to the case of constant coupling $G$ ( $B$-dependent $G$ ). We observe that, except for the $\eta^{\prime}$ mass, the $B$ dependence is rather mild in the case of the neutral mesons. On the other hand a rather
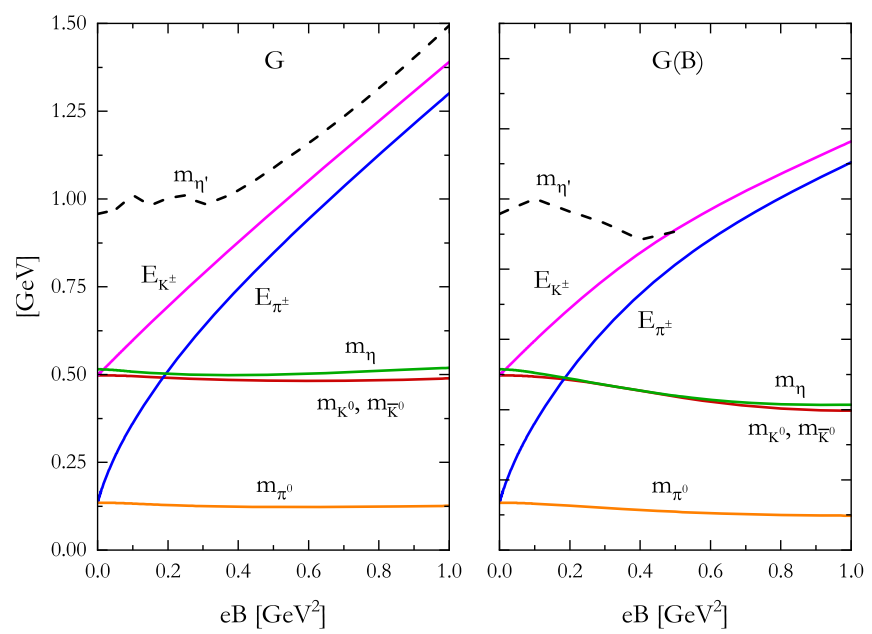

FIG. 3. Pseudoscalar neutral meson pole masses and charged mesons lowest energies as functions of $e B$ for constant (left) and $B$-dependent (right) coupling $G$.

strong increase with growing $B$ is found for charged meson masses. These results are analyzed in further detail in what follows.

The case of $\eta^{\prime}$ is somewhat special and, therefore, indicated in dashed lines in Fig. 3. In fact, already at $B=0$ its mass is above the threshold for $q \bar{q}$ decay and, thus, the associated $q \bar{q}$ polarization diagram receives an unphysical imaginary part. Following Ref. [73] we accept this as an unavoidable feature of the NJL model and define the $\eta^{\prime}$ mass as the real part of the corresponding pole in the complex plane. We should keep in mind, however, that this fact makes the predictions for the $\eta^{\prime}$ mass less reliable as compared to those of the other mesons. The situation worsens for finite magnetic field. First, new divergencies appear at low magnetic fields due the existence of thresholds associated with the Landau levels of the intermediate quark states. Although these divergencies are along the real axis, they originate the kind of oscillatory behavior found for $e B \lesssim 0.2 \mathrm{GeV}^{2}$. In passing, we note that including in the calculation the imaginary part of the polarization function makes these divergencies less harmful. If one neglects that contribution, as done in Ref. [74], the determination of $m_{\eta^{\prime}}$ becomes full of ambiguities making its determination even more troublesome. The other point has to do with the fact that at finite magnetic field the width is in general larger than the already non-negligible value at $B=0, \Gamma_{\eta^{\prime}}^{B=0}=269 \mathrm{MeV}$. For constant $G$, we encounter a nonmonotonic behavior of the width, which shows a closeto-vacuum mean value of $\Gamma_{\eta^{\prime}}^{B \text {,mean }}=332 \mathrm{MeV}$ but can reach values of $\Gamma_{\eta^{\prime}}^{B} \sim 590 \mathrm{MeV}$ at intermediate fields. On the other hand, for $B$-dependent $G$ the pace of growth of the width increases. At fields strengths around $e B \sim$ $0.5 \mathrm{GeV}^{2}$ the width exceeds the mass, with a value of $\Gamma_{\eta^{\prime}}^{B} \sim 1.46 \mathrm{GeV}$. This enhancement of the width, together 

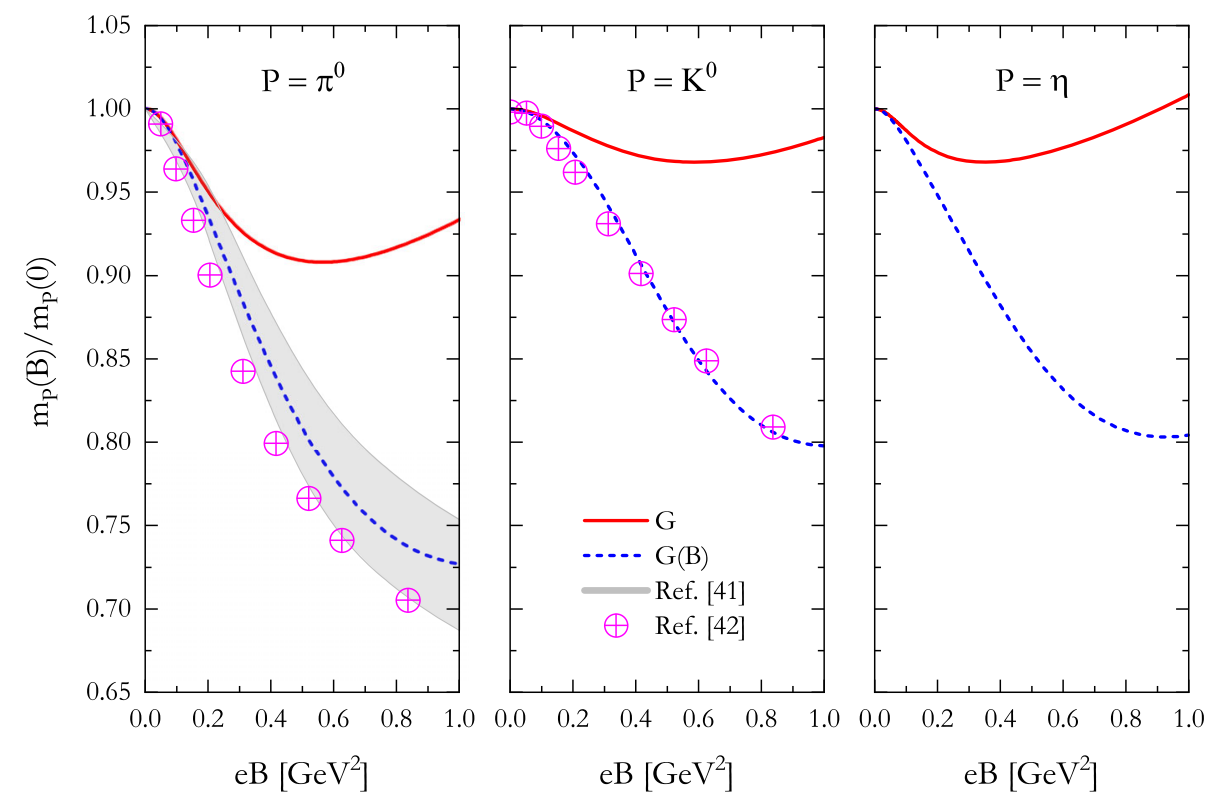

FIG. 4. Normalized neutral meson masses as functions of $e B$ for constant (red solid lines) and $B$-dependent (blue dashed lines) coupling G. LQCD results from Ref. [41] (gray band) and Ref. [42] (magenta circles) are added for comparison.

with the decrease of $G(B)$ as $B$ increases, results in the fact that for $e B \gtrsim 0.5 \mathrm{GeV}^{2}$ no solution of Eq. (36) can be found apart from the ones associated with $\pi^{0}$ and $\eta$. Namely, above such a value of the magnetic field the
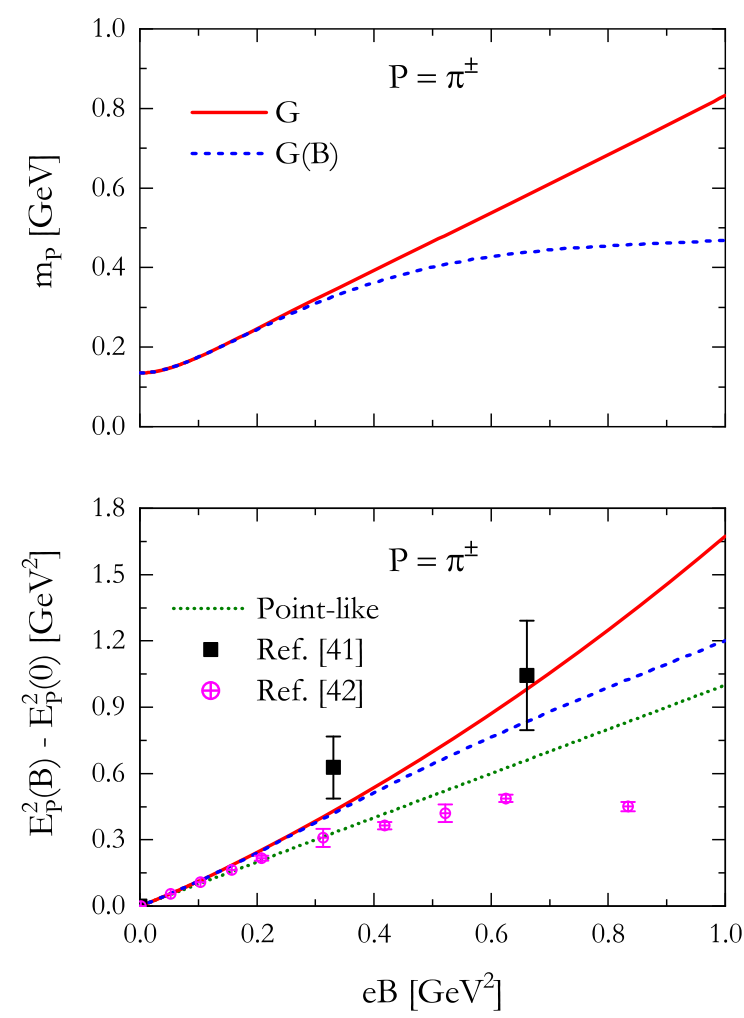

coupling strength is not enough to form an $\eta^{\prime}$ resonance in the $q \bar{q}$ continuum.

To discuss our results for the other neutral mesons $\left(\pi^{0}, K^{0}, \bar{K}^{0}\right.$ and $\left.\eta\right)$ in more detail we display in Fig. 4
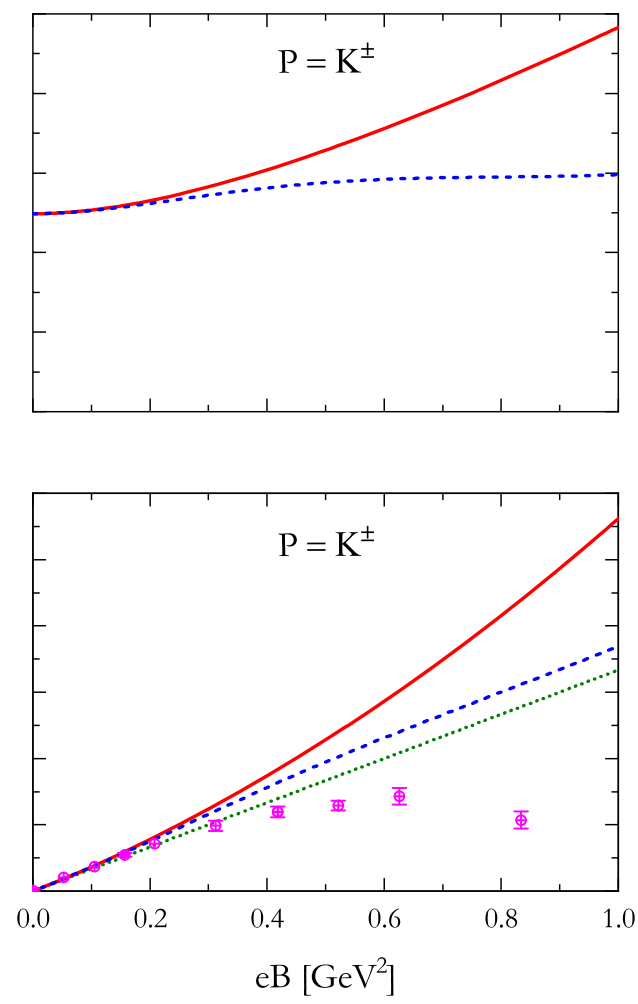

FIG. 5. Charged meson masses (top) and differences of squared lowest energies between the case at $B \neq 0$ and $B=0$ (bottom) for charged pions (left) and kaons (right) as a function of $e B$. Results for constant and $B$-dependent coupling $G$ are shown in red solid and blue dashed lines, respectively. Green dotted lines correspond to energies associated with pointlike charged mesons. LQCD results from Ref. [41] (black squares) and Ref. [42] (magenta circles) are added for comparison. 
the corresponding masses taken with respect to their values at $B=0$. We show results using a constant and a $B$-dependent coupling $G$ together with LCQD simulations from Refs. [41,42] for comparison. It should be noticed that these LQCD calculations correspond to nonphysical pion masses, i.e., 415 and $220 \mathrm{MeV}$, respectively, for vanishing magnetic fields. In both cases they point to a stronger decrease of the $\pi^{0}$ mass with increasing $B$ than the one found in our calculation with constant $G$. On the other hand, the results obtained using a $B$-dependent $G$ are in reasonable good agreement with LQCD ones. A similar observation have been made in Ref. [34] in the context of a two-flavor NJL model. This seems to also provide further support to the relation between the IMC effect and the reduction of the neutral pion mass at finite $B$ mentioned in Ref. [42]. In the case of $K^{0}$ and $\bar{K}^{0}$ masses (central panel), the only LQCD result that has been reported is that of Ref. [42]. We observe that, once again, a much better agreement with these results are obtained when a $B$-dependent coupling $G$ is used in the NJL model. Finally, in the right panel we show our predictions for the behavior of the normalized $\eta$-meson mass. They turn out to be quite similar to the ones obtained for the $K^{0}$ and $\bar{K}^{0}$ relative masses.

Finally, we consider the masses of charged pseudoscalar mesons $\pi^{ \pm}$and $K^{ \pm}$. In Fig. 5 we display the differences in their squared lowest energies from the case of a zero magnetic field, i.e., $E^{2}(B)-E^{2}(B=0)$. We also include their masses in the top graphs for completeness. We show results for $G$ and $G(B)$ as compared to a pointlike charged meson and LQCD simulations from Refs. [41,42]. We observe that for both charged pion and kaons our results show a stronger increase with growing $B$ as compared with the ones associated with pointlike mesons. Those obtained using a $B$-dependent $G$ are, however, somewhat closer to them. As for the comparison with LQCD results we note that in the case of charged pions there are significant differences between the results reported by the two different LQCD groups, specially at large magnetic fields. Although our results seem to be more consistent with those of Ref. [41] it should be recalled that they correspond to a larger (unphysical) value of the $B=0$ pion mass and have larger error bars. In any case, we see that, for both charged pions and kaons, our NJL results show no sign of the strong nonmonotonous behavior found in the LQCD calculation of Ref. [42]. Results obtained within the SU(2) version of the model [30], seem to indicate that the inclusion of quarks anomalous magnetic moments does not modify the trend of the charged pion mass obtained in the present work.

\section{CONCLUSIONS}

In this work we have considered the masses of the light pseudoscalar masses under the influence of strong magnetic fields in the framework of the SU(3) Nambu-JonaLasinio model that includes the 't Hooft-Maekawa flavor mixing interaction. The model parameters have been determined on fixing that for vanishing external field one reproduces the physical values of the $\pi, K$, and $\eta^{\prime}$ meson masses together with the pion weak decay constant. The possibility of using a magnetic field dependent fourfermion coupling constant in order to reproduce the inverse magnetic catalysis at finite temperature has also been considered. Since the NJL model is not renormalizable, the calculation of observables requires an appropriate regularization scheme in order to deal with ultraviolet divergences. Here we have used the magnetic field independent regularization procedure, in which only divergent vacuum contributions to quantities at zero external magnetic field are regularized. This scheme has been shown to provide more reliable predictions in comparison with other regularization methods often used in the literature [70].

At the mean field level, effective quark masses, shown in Fig. 1, steadily increase with $B$ for constant $G$ but display a nonmonotonous behavior for $G(B)$, which resembles the one found in Ref. [24]. Moreover, our results for the difference and average of the condensates calculated for both constant $G$ and $G(B)$ and their comparisons with the available LQCD results, as shown in Fig. 2, support the fact that the parametrizations used in this paper are in a very reasonable agreement with LQCD results.

In order to study meson masses we go beyond the meanfield approximation, considering second order corrections to the bosonized Euclidean action of the SU(3) NJL model. Mesons are treated as quantum fluctuations in the random phase approximation. While for neutral mesons one can take the usual momentum basis to diagonalize the corresponding polarization functions, this is not possible for charged mesons since Schwinger phases do not cancel out. In that case, we have employed a method based on the Ritus eigenfunction approach to magnetized relativistic systems. As discussed in Sec. II, at the quadratic level the inverse propagators corresponding to the neutral $\pi_{3}, \eta_{0}$, and $\eta_{8}$ fields are arranged in terms of a symmetric $3 \times 3$ matrix; the pole masses and widths of the physical mesons $\left(\pi^{0}, \eta\right.$ and $\eta^{\prime}$ ) are obtained as the roots of this inverse propagator matrix determinant. Note that in the $B \neq 0$ case, besides the 't Hooft-Maekawa interaction which breaks the $U_{A}(1)$ symmetry and is responsible for the coupling between the $\eta_{0}$ and $\eta_{8}$, the magnetic field also breaks the isospin symmetry, leading to a mixing between all three states [72]. This is in contrast to the $B=0$ case where due to the isospin symmetry $\left(M_{u}=M_{d}\right)$ the $\pi_{3}$ field is decoupled leaving only $\eta_{0}$ and $\eta_{8}$ mixed in a symmetric $2 \times 2$ matrix.

As already known from the usual SU(3) NJL model at $B=0$, the $\eta^{\prime}$ meson comes out in the model as a resonance or unstable particle. In this case, the propagator becomes a complex number and from the analysis of the complex pole, the mass of the resonance is obtained. In the presence of a finite magnetic field, the situation is more dramatic since the propagators may develop several poles depending on $B$, which have to be properly treated. We have 
developed in this paper a new formalism to deal with this situation. Of course, the results for the $\eta^{\prime}$ meson are less reliable and its calculation certainly approaches the limit of applicability of the NJL model, since this model does not include confinement. In fact, we find that using $G(B)$ the coupling strength is not enough to form an $\eta^{\prime}$ resonance for $e B \gtrsim 0.5 \mathrm{GeV}^{2}$.

Our results for the normalized $\pi^{0}$ mass in Fig. 4 show that, for constant $G$, the mass displays a non-monotonous behavior with $B$, which initially decreases but is afterwards enhanced for $e B \gtrsim 0.5 \mathrm{GeV}^{2}$. On the other hand, using a $B$-dependent coupling $G(B)$ we recover the monotonous decreasing behavior found in LQCD results. Something similar happens with $K^{0}$ and $\bar{K}^{0}$ masses. For $\eta$, our prediction is similar to that of $K^{0}$ and $\bar{K}^{0}$. We thus conclude that incorporating the inverse magnetic catalysis in the NJL model, here through the $G(B)$ coupling, is fundamental for qualitatively reproduce the available LQCD results. Concerning charged mesons, our results for the differences in their squared lowest energies from the $B=0$ case are shown in Fig. 5, where a strong enhancement with $B$ is seen. This increase even surpasses the one associated with a pointlike charged meson. Our NJL results are in reasonable agreement with LQCD results of Ref. [41] within error bars. On the other hand, no sign of the nonmonotonous behavior found in the LQCD calculation of Ref. [42] is observed.

obtains

$$
c_{f f^{\prime}}^{0}\left(q^{2}\right)=\frac{N_{c}}{2 \pi^{2}} \int_{0}^{\infty} d z \int_{0}^{1} d y \exp \left\{-z\left[y M_{f}^{2}+(1-y) M_{f^{\prime}}^{2}+y(1-y) q^{2}-i \epsilon\right]\right\} \frac{1}{z}\left[M_{f} M_{f^{\prime}}+\frac{2}{z}-y(1-y) q^{2}\right] .
$$

We have expressed this function in the proper time formalism. Through some algebraic manipulation, it can also be written in the following standard form

$$
c_{f f^{\prime}}^{0}\left(q^{2}\right)=2 N_{c}\left\{\frac{I_{1 f}^{0}+I_{1 f^{\prime}}^{0}}{2}+\left[q^{2}+\left(M_{f}-M_{f^{\prime}}\right)^{2}\right] I_{2 f f^{\prime}}^{0}\left(q^{2}\right)\right\}
$$

where the integrals $I_{1 f}^{0}$ and $I_{2 f f^{\prime}}^{0}$ are defined by

$$
\begin{aligned}
I_{1 f}^{0} & =4 \int_{p} \frac{1}{p^{2}+M_{f}^{2}}, \\
I_{2 f f^{\prime}}^{0}\left(q^{2}\right) & =-2 \int_{p} \frac{1}{\left(p_{-}^{2}+M_{f}^{2}-i \epsilon\right)\left(p_{+}^{2}+M_{f^{\prime}}^{2}-i \epsilon\right)} .
\end{aligned}
$$

In order to regularize the vacuum loop integrals we introduce a $3 \mathrm{D}$ cutoff $\Lambda$. For $I_{1 f}^{0}$ one gets the regularized function

$$
I_{1 f}^{\mathrm{vac}}=\frac{1}{2 \pi^{2}}\left[\Lambda \sqrt{M_{f}^{2}+\Lambda^{2}}+M_{f}^{2} \ln \left(\frac{M_{f}}{\Lambda+\sqrt{M_{f}^{2}+\Lambda^{2}}}\right)\right] .
$$


For $I_{2 f f^{\prime}}^{0}\left(q^{2}\right)$ we note that in order to determine the meson masses, the external momenta $q$ in the loop integrals has to be extended to the region $q^{2}<0$. Hence, we find it convenient to introduce $q^{2}=-q_{m}^{2}$, with $q_{m}>0$. In this case the function has several poles. To treat them, we go from Euclidean to the original Minkowski space by taking $p_{4}=-i p_{0}$. Then, by choosing appropriate contours the $p_{0}$ integral can be calculated in the complex plane to yield

$$
I_{2 f f^{\prime}}^{\mathrm{vac}}\left(q^{2}\right)=-\frac{1}{8 \pi^{2} q_{m}^{2}} \int_{0}^{\Lambda} d p \frac{p^{2}}{p^{2}-r-i \epsilon}\left[\frac{q_{m}^{2}+M_{f}^{2}-M_{f^{\prime}}^{2}}{\sqrt{p^{2}+M_{f}^{2}}}+\frac{q_{m}^{2}-M_{f}^{2}+M_{f^{\prime}}^{2}}{\sqrt{p^{2}+M_{f^{\prime}}^{2}}}\right],
$$

where

$$
r=\frac{1}{4 q_{m}^{2}}\left[\left(M_{f}-M_{f^{\prime}}\right)^{2}-q_{m}^{2}\right]\left[\left(M_{f}+M_{f^{\prime}}\right)^{2}-q_{m}^{2}\right]
$$

Depending on the value of $q_{m}$, this expression may still have a pole in a point of the integration line if $r>0$. For those regions of $q_{m}$ where a pole exists, we proceed by employing a generalized version of the Sokhotski-Plemelj formula. Assuming there exists a function $f(x)$ that has single poles at a set of values $x_{j}$, for which exist two other functions $g(x)$ and $h(x)$ such that $g\left(x_{j}\right) \neq 0$ and $h\left(x_{j}\right) \neq 0$, then

$$
\lim _{\epsilon \rightarrow 0^{+}} \int_{a}^{b} d x \frac{h(x)}{f(x)+i \epsilon g(x)}=\mathrm{PV} \int_{a}^{b} d x \frac{h(x)}{f(x)}-i \pi \sum_{j} \frac{h\left(x_{j}\right)}{\left|f^{\prime}\left(x_{j}\right)\right|} \operatorname{sign}\left[g\left(x_{j}\right)\right],
$$

where PV denotes the Cauchy principal value of the integral. By using this property we can fully calculate the complex function $I_{2 f f^{\prime}}^{\mathrm{vac}}$ in the most general case. For the regularized real part we get

$\operatorname{Re}\left[I_{2 f f^{\prime}}^{\mathrm{vac}}\left(-q_{m}^{2}\right)\right]=-\frac{1}{8 \pi^{2} q_{m}^{2}}\left\{\left(q_{m}^{2}+M_{f}^{2}-M_{f^{\prime}}^{2}\right)\left[\operatorname{arcsinh}\left(\frac{\Lambda}{M_{f}}\right)-F_{f}\right]+\left(q_{m}^{2}-M_{f}^{2}+M_{f^{\prime}}^{2}\right)\left[\operatorname{arcsinh}\left(\frac{\Lambda}{M_{f^{\prime}}}\right)-F_{f^{\prime}}\right]\right\}$,

where

$$
F_{f}= \begin{cases}\frac{y_{+}}{\sqrt{M_{f}^{2}+y_{+}^{2}}} \operatorname{arctanh}\left(\frac{\Lambda}{y_{+}} \sqrt{\frac{M_{f}^{2}+y_{+}^{2}}{M_{f}^{2}+\Lambda^{2}}}\right) & \text { for } q_{m}<q_{m}^{(0)} \text { or } q_{m}>q_{m}^{(3)} \\ \frac{y_{+}}{\sqrt{M_{f}^{2}+y_{+}^{2}}} \operatorname{arccoth}\left(\frac{\Lambda}{y_{+}} \sqrt{\frac{M_{f}^{2}+y_{+}^{2}}{M_{f}^{2}+\Lambda^{2}}}\right) & \text { for } q_{m}^{(0)}<q_{m}<q_{m}^{(1)} \text { or } q_{m}^{(2)}<q_{m}<q_{m}^{(3)} \\ \frac{y_{-}}{\sqrt{M_{f}^{2}-y_{-}^{2}}} \arctan \left(\frac{\Lambda}{y_{-}} \sqrt{\frac{M_{f}^{2}-y_{-}^{2}}{M_{f}^{2}+\Lambda^{2}}}\right) & \text { for } q_{m}^{(1)}<q_{m}<q_{m}^{(2)}\end{cases}
$$

Here $y_{ \pm}=\sqrt{ \pm r}$, with $r$ defined in Eq. (A7), and

$$
q_{m}^{\left(\frac{0}{3}\right)}=\left[M_{f}^{2}+M_{f^{\prime}}^{2}+2 \Lambda^{2} \mp 2 \sqrt{\left(\Lambda^{2}+M_{f}^{2}\right)\left(\Lambda^{2}+M_{f^{\prime}}^{2}\right)}\right]^{1 / 2}, \quad q_{m}^{\left(\frac{1}{2}\right)}=\left|M_{f} \mp M_{f^{\prime}}\right| .
$$

For the regularized imaginary part we get

$$
\operatorname{Im}\left[I_{2 f f^{\prime}}^{\mathrm{vac}}\left(-q_{m}^{2}\right)\right]=\left\{\begin{array}{ll}
-\frac{y_{+}}{4 \pi q_{m}} & \text { for } q_{m}^{(2)}<q_{m}<q_{m}^{(3)} \\
0 & \text { otherwise }
\end{array} .\right.
$$

Putting all together, the regularized version of the vacuum $c_{f f^{\prime}}^{0}$ function defined in Eq. (A3) is given by

$$
c_{f f^{\prime}}^{\mathrm{vac}}\left(q^{2}=-q_{m}^{2}\right)=2 N_{c}\left\{\frac{I_{1 f}^{\mathrm{vac}}+I_{1 f^{\prime}}^{\mathrm{vac}}}{2}-\left[q_{m}^{2}-\left(M_{f}-M_{f^{\prime}}\right)^{2}\right] I_{2 f f^{\prime}}^{\mathrm{vac}}\left(-q_{m}^{2}\right)\right\} .
$$




\section{APPENDIX B: EXPLICIT EXPRESSION OF THE NEUTRAL MAGNETIC FUNCTION $c_{f f^{\prime}}^{\mathrm{mag}}\left(q_{\perp}^{2}, q_{\|}^{2}\right)$}

The unregularized neutral function $c_{f f^{\prime}}$ in momentum space was originally defined in Eq. (25). Following a standard calculation (see [59] for details) and assuming $Q_{f}=Q_{f^{\prime}}$ we obtain

$$
\begin{aligned}
c_{f f^{\prime}}\left(q_{\perp}^{2}, q_{\|}^{2}\right)= & \frac{N_{c} B_{f}}{2 \pi^{2}} \int_{0}^{\infty} d z \int_{0}^{1} d y \exp \left\{-z\left[y M_{f}^{2}+(1-y) M_{f^{\prime}}^{2}+y(1-y) q_{\|}^{2}-i \epsilon\right]\right\} \\
& \times \exp \left[-\frac{q_{\perp}^{2}}{B_{f}} \gamma_{f}(y, z)\right]\left\{\left[M_{f} M_{f^{\prime}}+\frac{1}{z}-y(1-y) q_{\|}^{2}\right] \operatorname{coth}\left(z B_{f}\right)+\frac{B_{f}}{\sinh ^{2}\left(z B_{f}\right)}\left[1-\frac{q_{\perp}^{2}}{B_{f}} \gamma_{f}(y, z)\right]\right\},
\end{aligned}
$$

where

$$
\gamma_{f}(y, z)=\frac{\sinh \left(y z B_{f}\right) \sinh \left[(1-y) z B_{f}\right]}{\sinh \left(z B_{f}\right)}
$$

As usual, here we have used the changes of variables $\tau=y z$ and $\tau^{\prime}=(1-y) z, \tau$ and $\tau^{\prime}$ being the integration parameters associated with the quark propagators as in Eq. (7). The $B \rightarrow 0$ limit of this expression $c_{f f^{\prime}}^{0}$ is given by Eq. (A2). Then, the finite magnetic contribution is defined within the MFIR scheme as the difference

$$
c_{f f^{\prime}}^{\mathrm{mag}}\left(q_{\perp}^{2}, q_{\|}^{2}\right) \equiv c_{f f^{\prime}}\left(q_{\perp}^{2}, q_{\|}^{2}\right)-c_{f f^{\prime}}^{0}\left(q^{2}\right) .
$$

For the calculation of the meson masses we can take $q_{\perp}^{2}=0$ and $q_{\|}^{2}=-q_{m}^{2}$, with $q_{m}>0$. Assuming that $q_{m}<M_{f}+M_{f^{\prime}}$, one can integrate by parts to write this function in the form

$$
c_{f f^{\prime}}^{\mathrm{mag}}\left(q_{\perp}^{2}=0, q_{\|}^{2}=-q_{m}^{2}\right)=2 N_{c}\left\{\frac{I_{1 f}^{\mathrm{mag}}+I_{1 f^{\prime}}^{\mathrm{mag}}}{2}-\left[q_{m}^{2}-\left(M_{f}-M_{f^{\prime}}\right)^{2}\right] I_{2 f f^{\prime}}^{\mathrm{mag}}\left(-q_{m}^{2}\right)\right\},
$$

where the integral $I_{1 f}^{\mathrm{mag}}$ is defined as

$$
\begin{aligned}
I_{1 f}^{\mathrm{mag}} & =\frac{B_{f}}{4 \pi^{2}} \int_{0}^{\infty} \frac{d z}{z} e^{-2 z x_{f}}\left(\operatorname{coth} z-\frac{1}{z}\right), \\
& =\frac{B_{f}}{2 \pi^{2}}\left[\ln \Gamma\left(x_{f}\right)-\left(x_{f}-\frac{1}{2}\right) \ln x_{f}+x_{f}-\frac{\ln 2 \pi}{2}\right],
\end{aligned}
$$

where $x_{f}=M_{f}^{2} /\left(2 B_{f}\right)$. On the other hand $I_{2 f f^{\prime}}^{\mathrm{mag}}$ is given by

$$
I_{2 f f^{\prime}}^{\mathrm{mag}}\left(-q_{m}^{2}\right)=-\frac{1}{8 \pi^{2}} \int_{0}^{1} d y \int_{0}^{\infty} d z e^{-2 z\left(\bar{x}_{f f^{\prime}}-i \epsilon\right)}\left(\operatorname{coth} z-\frac{1}{z}\right)
$$

with

$$
x_{f f^{\prime}}=\frac{y M_{f}^{2}+(1-y) M_{f^{\prime}}^{2}-y(1-y) q_{m}^{2}}{2 B_{f}} .
$$

When $q_{m}<M_{f}+M_{f^{\prime}}$ we always have that $x_{f f^{\prime}}>0$. Then function $I_{2 f f^{\prime}}^{\mathrm{mag}}$ as given in Eq. (B6) is well defined and can alternatively written as

$$
I_{2 f f^{\prime}}^{\mathrm{mag}}\left(-q_{m}^{2}\right)=\frac{1}{8 \pi^{2}} \int_{0}^{1} d y\left[\psi\left(\bar{x}_{f f^{\prime}}-i \epsilon\right)-\ln \left(\bar{x}_{f f^{\prime}}-i \epsilon\right)+\frac{1}{2\left(\bar{x}_{f f^{\prime}}-i \epsilon\right)}\right]
$$

where $\psi(x)$ is the digamma function. Note that in this case one can safely take the $\epsilon \rightarrow 0$ limit.

On the other hand, when $q_{m}>M_{f}+M_{f^{\prime}}$ it happens that $\bar{x}_{f f^{\prime}}$ can be negative in the integration domain. In this case, the integral in Eq. (B6) is not convergent. However, one can still proceed by considering the analytic extension of the form 
given in Eq. (B8). Since $\bar{x}_{f f^{\prime}}$ is a positive quadratic function of $y$, it is immediate to see that $\psi\left(\bar{x}_{f f^{\prime}}\right)$ has $N+1$ poles, where

$$
N=\text { Floor }\left\{\frac{1}{2 B_{f}}\left[1-\left(\frac{M_{f}-M_{f^{\prime}}}{q_{m}}\right)^{2}\right]\left[\frac{q_{m}^{2}}{4}-\left(\frac{M_{f}+M_{f^{\prime}}}{2}\right)^{2}\right]\right\}
$$

To proceed we first isolate the poles by using the digamma recurrence relation

$$
\psi\left(\bar{x}_{f f^{\prime}}-i \epsilon\right)=\psi\left(\bar{x}_{f f^{\prime}}+N+1\right)-\sum_{n=0}^{N} \frac{1}{\bar{x}_{f f^{\prime}}+n-i \epsilon} .
$$

Expressed this way, the first term in the right-hand side is pole free. Then

$$
I_{2 f f^{\prime}}^{\mathrm{mag}}\left(-q_{m}^{2}\right)=\frac{1}{8 \pi^{2}} \int_{0}^{1} d y\left[\psi\left(\bar{x}_{f f^{\prime}}+N+1\right)-\ln \left(\bar{x}_{f f^{\prime}}-i \epsilon\right)-\frac{1}{2} \sum_{n=0}^{N} \frac{g_{n}}{\bar{x}_{f f^{\prime}}+n-i \epsilon}\right],
$$

where $g_{n}=2-\delta_{n 0}$. The complex logarithm is defined by taking the principal branch. For the region where $\bar{x}_{f f^{\prime}}<0$ we have

$$
\lim _{\epsilon \rightarrow 0} \ln \left(-\left|\bar{x}_{f f^{\prime}}\right|-i \epsilon\right)=\ln \left(\left|\bar{x}_{f f^{\prime}}\right|\right)-i \pi
$$

Lastly, the third term on the right-hand side of Eq. (B11) contains two simple poles, which once again can be handled using the generalization of the Sokhotski-Plemelj formula presented in Eq. (A8). After some algebra we finally obtain that for $q_{m}>M_{f}+M_{f^{\prime}}$

$$
\begin{aligned}
I_{2 f f^{\prime}}^{\mathrm{mag}}\left(q_{\|}^{2}=-q_{m}^{2}\right)= & -\frac{1}{8 \pi^{2}}\left\{\ln \left[\frac{\left(M_{f}\right)^{1-\alpha}\left(M_{f^{\prime}}\right)^{1+\alpha}}{2 B_{f}}\right]+\frac{\beta_{0}}{2} \ln \left[\frac{\alpha^{2}-\left(1+\beta_{0}\right)^{2}}{\alpha^{2}-\left(1-\beta_{0}\right)^{2}}\right]-2+\frac{B_{f}}{q_{m}^{2}} \sum_{n=0}^{N} \frac{g_{n}}{\beta_{n}} \ln \left[\frac{\alpha^{2}-\left(1-\beta_{n}\right)^{2}}{\alpha^{2}-\left(1+\beta_{n}\right)^{2}}\right]\right\} \\
& +\frac{1}{8 \pi^{2}} \int_{0}^{1} d y \psi\left(\bar{x}_{f f^{\prime}}+N+1\right)+\frac{i}{8 \pi}\left(\beta_{0}-\frac{2 B_{f}}{q_{m}^{2}} \sum_{n=0}^{N} \frac{g_{n}}{\beta_{n}}\right),
\end{aligned}
$$

with

$$
\alpha=\frac{M_{f^{\prime}}^{2}-M_{f}^{2}}{q_{m}^{2}}, \quad \beta_{n}=\sqrt{\left[1-\left(\frac{M_{f^{\prime}}-M_{f}}{q_{m}}\right)^{2}\right]\left[1-\left(\frac{M_{f^{\prime}}+M_{f}}{q_{m}}\right)^{2}\right]-\frac{8 n B_{f}}{q_{m}^{2}}} .
$$

We remark that the calculation of $I_{2 f f^{\prime}}^{\mathrm{mag}}$ was performed here within the proper time formalism, which is well defined for $q_{m}<M_{f}+M_{f^{\prime}}$ and leads to Eq. (B8). For $q_{m}>M_{f}+M_{f^{\prime}}$ we have taken the analytic continuation of this equation. As a consistency check, we have repeated the calculation using the Landau level representation for the quark propagator in Minkowski space, which is well defined for all $q_{m}$, obtaining the same final result of Eq. (B13).

[1] K. Tuchin, Particle production in strong electromagnetic fields in relativistic heavy-ion collisions, Adv. High Energy Phys. 2013, 490495 (2013).

[2] D. Grasso and H. Rubinstein, Magnetic fields in the early universe, Phys. Rep. 348, 163 (2001).

[3] R. C. Duncan and C. Thompson, Formation of very strongly magnetized neutron stars: Implications for gamma-ray bursts, Astrophys. J. Lett. 392, L9 (1992).
[4] K. Fukushima, Views of the chiral magnetic effect, Lect. Notes Phys. 871, 241 (2013).

[5] D. E. Kharzeev, The chiral magnetic effect and anomalyinduced transport, Prog. Part. Nucl. Phys. 75, 133 (2014).

[6] W. Li and G. Wang, Chiral magnetic effects in nuclear collisions, Annu. Rev. Nucl. Part. Sci. 70, 293 (2020). 
[7] E. Gorbar, V. Miransky, I. Shovkovy, and X. Wang, Radiative corrections to chiral separation effect in QED, Phys. Rev. D 88, 025025 (2013).

[8] D. Kharzeev and H. Yee, Chiral magnetic wave, Phys. Rev. D 83, 085007 (2011).

[9] I. Shovkovy, D. Rybalka, and E. Gorbar, The overdamped chiral magnetic wave, Proc. Sci., Confinement2018 (2018) 029 [arXiv:1811.10635].

[10] Y. Burnier, D. Kharzeev, J. Liao, and H. Yee, Chiral Magnetic Wave at Finite Baryon Density and the Electric Quadrupole Moment of Quark-Gluon Plasma in Heavy Ion Collisions, Phys. Rev. Lett. 107, 052303 (2011).

[11] H. Yee and Y. Yin, Realistic implementation of chiral magnetic wave in heavy ion collisions, Phys. Rev. C 89, 044909 (2014).

[12] L. Adamczyk et al. (STAR Collaboration), Observation of Charge Asymmetry Dependence of Pion Elliptic Flow and the Possible Chiral Magnetic Wave in Heavy-Ion Collisions, Phys. Rev. Lett. 114, 252302 (2015).

[13] K. Fukushima, D. Kharzeev, and H. Warringa, The chiral magnetic effect, Phys. Rev. D 78, 074033 (2008).

[14] D. Kharzeev, J. Liao, S. Voloshin, and G. Wang, Chiral magnetic and vortical effects in high-energy nuclear collisions-A status report, Prog. Part. Nucl. Phys. 88, 1 (2016).

[15] M. D'Elia, S. Mukherjee, and F. Sanfilippo, QCD phase transition in a strong magnetic background, Phys. Rev. D 82, 051501 (2010).

[16] J. Andersen, W. Naylor, and A. Tranberg, Phase diagram of QCD in a magnetic field: A review, Rev. Mod. Phys. 88, 025001 (2016).

[17] V. Miransky and I. Shovkovy, Quantum field theory in a magnetic field: From quantum chromodynamics to graphene and Dirac semimetals, Phys. Rep. 576, 1 (2015).

[18] G. Bali, F. Bruckmann, G. Endrődi, Z. Fodor, S. Katz, S. Krieg, A. Schafer, and K. Szabo, The QCD phase diagram for external magnetic fields, J. High Energy Phys. 02 (2012) 044.

[19] G. Bali, F. Bruckmann, G. Endrődi, Z. Fodor, S. Katz, and A. Schäfer, QCD quark condensate in external magnetic fields, Phys. Rev. D 86, 071502 (2012).

[20] G. Bali, F. Bruckmann, G. Endrődi, S. Katz, and A. Schäfer, The QCD equation of state in background magnetic fields, J. High Energy Phys. 08 (2014) 177.

[21] V. Bornyakov, P. Buividovich, N. Cundy, O. Kochetkov, and A. Schäfer, Deconfinement transition in two-flavor lattice QCD with dynamical overlap fermions in an external magnetic field, Phys. Rev. D 90, 034501 (2014).

[22] A. Bandyopadhyay and R. Farias, Inverse magnetic catalysis-How much do we know about?, Eur. Phys. J. Special Topics 230, 719 (2021).

[23] M. D’Elia, F. Manigrasso, F. Negro, and F. Sanfilippo, QCD phase diagram in a magnetic background for different values of the pion mass, Phys. Rev. D 98, 054509 (2018).

[24] G. Endrődi, M. Giordano, S. D. Katz, T. Kovács, and F. Pittler, Magnetic catalysis and inverse catalysis for heavy pions, J. High Energy Phys. 07 (2019) 007.

[25] J. Andersen, QCD phase diagram in a constant magnetic background: Inverse magnetic catalysis: where models meet the lattice, Eur. Phys. J. A 57, 189 (2021).
[26] S. Mao, Inverse magnetic catalysis in Nambu-Jona-Lasinio model beyond mean field, Phys. Lett. B 758, 195 (2016).

[27] S. Fayazbakhsh and N. Sadooghi, Anomalous magnetic moment of hot quarks, inverse magnetic catalysis, and reentrance of the chiral symmetry broken phase, Phys. Rev. D 90, 105030 (2014).

[28] J. Mei and S. Mao, Inverse catalysis effect of the quark anomalous magnetic moment to chiral restoration and deconfinement phase transitions, Phys. Rev. D 102, 114035 (2020).

[29] N. Chaudhuri, S. Ghosh, S. Sarkar, and P. Roy, Effects of quark anomalous magnetic moment on the thermodynamical properties and mesonic excitations of magnetized hot and dense matter in PNJL model, Eur. Phys. J. A 56, 213 (2020).

[30] K. Xu, J. Chao, and M. Huang, Effect of the anomalous magnetic moment of quarks on magnetized QCD matter and meson spectra, Phys. Rev. D 103, 076015 (2021).

[31] M. Ferreira, P. Costa, O. Lourenço, T. Frederico, and C. Providência, Inverse magnetic catalysis in the $(2+1)$-flavor Nambu-Jona-Lasinio and Polyakov-Nambu-Jona-Lasinio models, Phys. Rev. D 89, 116011 (2014).

[32] R. Farias, K. Gomes, G. Krein, and M. Pinto, Importance of asymptotic freedom for the pseudocritical temperature in magnetized quark matter, Phys. Rev. C 90, 025203 (2014).

[33] R. Farias, V. Timóteo, S. Avancini, M. Pinto, and G. Krein, Thermo-magnetic effects in quark matter: Nambu-JonaLasinio model constrained by lattice QCD, Eur. Phys. J. A 53, 101 (2017).

[34] S. Avancini, R. Farias, M. Pinto, W. Tavares, and V. Timóteo, $\pi_{0}$ pole mass calculation in a strong magnetic field and lattice constraints, Phys. Lett. B 767, 247 (2017).

[35] S. Avancini, R. Farias, and W. Tavares, Neutral meson properties in hot and magnetized quark matter: a new magnetic field independent regularization scheme applied to NJL-type model, Phys. Rev. D 99, 056009 (2019).

[36] S. Avancini, R. Farias, M. Pinto, T. Restrepo, and W. Tavares, Regularizing thermo and magnetic contributions within nonrenormalizable theories, Phys. Rev. D 103, 056009 (2021).

[37] G. Endrődi and G. Markó, Magnetized baryons and the QCD phase diagram: NJL model meets the lattice, J. High Energy Phys. 08 (2019) 036.

[38] V. Pagura, D. Gomez Dumm, S. Noguera, and N. Scoccola, Magnetic catalysis and inverse magnetic catalysis in nonlocal chiral quark models, Phys. Rev. D 95, 034013 (2017).

[39] D. G. Dumm, M. I. Villafañe, S. Noguera, V. Pagura, and N. Scoccola, Strong magnetic fields in nonlocal chiral quark models, Phys. Rev. D 96, 114012 (2017).

[40] E. Luschevskaya, O. Solovjeva, and O. Teryaev, Magnetic polarizability of pion, Phys. Lett. B 761, 393 (2016).

[41] G. Bali, B. Brandt, G. Endrődi, and B. Gläßle, Meson masses in electromagnetic fields with Wilson fermions, Phys. Rev. D 97, 034505 (2018).

[42] H.-T. Ding, S.-T. Li, A. Tomiya, X.-D. Wang, and Y. Zhang, Chiral properties of $(2+1)$-flavor QCD in strong magnetic fields at zero temperature, Phys. Rev. D 104, 014505 (2021).

[43] N. Agasian and I. Shushpanov, Gell-Mann-Oakes-Renner relation in a magnetic field at finite temperature, J. High Energy Phys. 10 (2001) 006. 
[44] J. Andersen, Chiral perturbation theory in a magnetic background-finite-temperature effects, J. High Energy Phys. 10 (2012) 005.

[45] V. Orlovsky and Y. Simonov, Nambu-Goldstone mesons in strong magnetic field, J. High Energy Phys. 09 (2013) 136.

[46] G. Colucci, E. Fraga, and A. Sedrakian, Chiral pions in a magnetic background, Phys. Lett. B 728, 19 (2014).

[47] A. Ayala, R. Farias, S. Hernández-Ortiz, L. Hernández, D. M. Paret, and R. Zamora, Magnetic field-dependence of the neutral pion mass in the linear sigma model coupled to quarks: The weak field case, Phys. Rev. D 98, 114008 (2018).

[48] A. Das and N. Haque, Neutral pion mass in the linear sigma model coupled to quarks at arbitrary magnetic field, Phys. Rev. D 101, 074033 (2020).

[49] A. Ayala, J. Hernández, L. A. Hernández, R. Farias, and R. Zamora, Magnetic field dependence of the neutral pion mass in the linear sigma model with quarks: The strong field case, Phys. Rev. D 103, 054038 (2021).

[50] K. Kamikado and T. Kanazawa, Chiral dynamics in a magnetic field from the functional renormalization group, J. High Energy Phys. 03 (2014) 009.

[51] M. Andreichikov, B. Kerbikov, E. Luschevskaya, Y. Simonov, and O. Solovjeva, The evolution of meson masses in a strong magnetic field, J. High Energy Phys. 05 (2017) 007.

[52] Y. Simonov, Pion decay constants in a strong magnetic field, Phys. At. Nucl. 79, 455 (2016).

[53] M. Andreichikov and Y. Simonov, Chiral physics in the magnetic field with quark confinement contribution, Eur. Phys. J. C 78, 902 (2018).

[54] C. Dominguez, M. Loewe, and C. Villavicencio, QCD determination of the magnetic field dependence of QCD and hadronic parameters, Phys. Rev. D 98, 034015 (2018).

[55] S. Fayazbakhsh, S. Sadeghian, and N. Sadooghi, Properties of neutral mesons in a hot and magnetized quark matter, Phys. Rev. D 86, 085042 (2012).

[56] S. Fayazbakhsh and N. Sadooghi, Weak decay constant of neutral pions in a hot and magnetized quark matter, Phys. Rev. D 88, 065030 (2013).

[57] S. Avancini, W. Tavares, and M. Pinto, Properties of magnetized neutral mesons within a full RPA evaluation, Phys. Rev. D 93, 014010 (2016).

[58] M. Coppola, D. G. Dumm, and N. Scoccola, Charged pion masses under strong magnetic fields in the NJL model, Phys. Lett. B 782, 155 (2018).
[59] M. Coppola, D. G. Dumm, S. Noguera, and N. Scoccola, Neutral and charged pion properties under strong magnetic fields in the NJL model, Phys. Rev. D 100, 054014 (2019).

[60] R. Zhang, W.-J. Fu, and Y.-X. Liu, Properties of mesons in a strong magnetic field, Eur. Phys. J. C 76, 307 (2016).

[61] S. Mao and Y. Wang, Effect of discrete quark momenta on the Goldstone mode in a magnetic field, Phys. Rev. D 96, 034004 (2017).

[62] S. Mao, Pions in magnetic field at finite temperature, Phys. Rev. D 99, 056005 (2019).

[63] Z. Wang and P. Zhuang, Meson properties in magnetized quark matter, Phys. Rev. D 97, 034026 (2018).

[64] H. Liu, X. Wang, L. Yu, and M. Huang, Neutral and charged scalar mesons, pseudoscalar mesons, and diquarks in magnetic fields, Phys. Rev. D 97, 076008 (2018).

[65] D. G. Dumm, M. I. Villafañe, and N. Scoccola, Neutral meson properties under an external magnetic field in nonlocal chiral quark models, Phys. Rev. D 97, 034025 (2018).

[66] D. G. Dumm, M. I. Villafañe, and N. Scoccola, Properties of magnetized neutral pions at zero and finite temperature in nonlocal chiral quark models, Phys. Rev. D 101, 116018 (2020).

[67] K. Hattori, T. Kojo, and N. Su, Mesons in strong magnetic fields: (I) General analyses, Nucl. Phys. A951, 1 (2016).

[68] T. Kojo, Neutral and charged mesons in magnetic fields: A resonance gas in a non-relativistic quark model, arXiv: 2104.00376

[69] A. Mishra and S. Misra, Strange mesons in strong magnetic fields, Int. J. Mod. Phys. E 30, 2150014 (2021).

[70] S. Avancini, R. Farias, N. Scoccola, and W. Tavares, NJL-type models in the presence of intense magnetic fields: The role of the regularization prescription, Phys. Rev. D 99, 116002 (2019).

[71] V. I. Ritus, Method of eigenfunctions and mass operator in quantum electrodynamics of a constant field, Sov. Phys. JETP 48, 788 (1978).

[72] G. Cao, Recent progresses on QCD phases in a strong magnetic field-Views from Nambu-Jona-Lasinio model, Eur. Phys. J. A 57, 264 (2021).

[73] P. Rehberg, S. Klevansky, and J. Hufner, Hadronization in the SU(3) Nambu-Jona-Lasinio model, Phys. Rev. C 53, 410 (1996).

[74] T. Hatsuda and T. Kunihiro, QCD phenomenology based on a chiral effective Lagrangian, Phys. Rep. 247, 221 (1994). 